\title{
Tadr Is an Axonal Histidine Transporter Required for Visual
} Neurotransmission in Drosophila

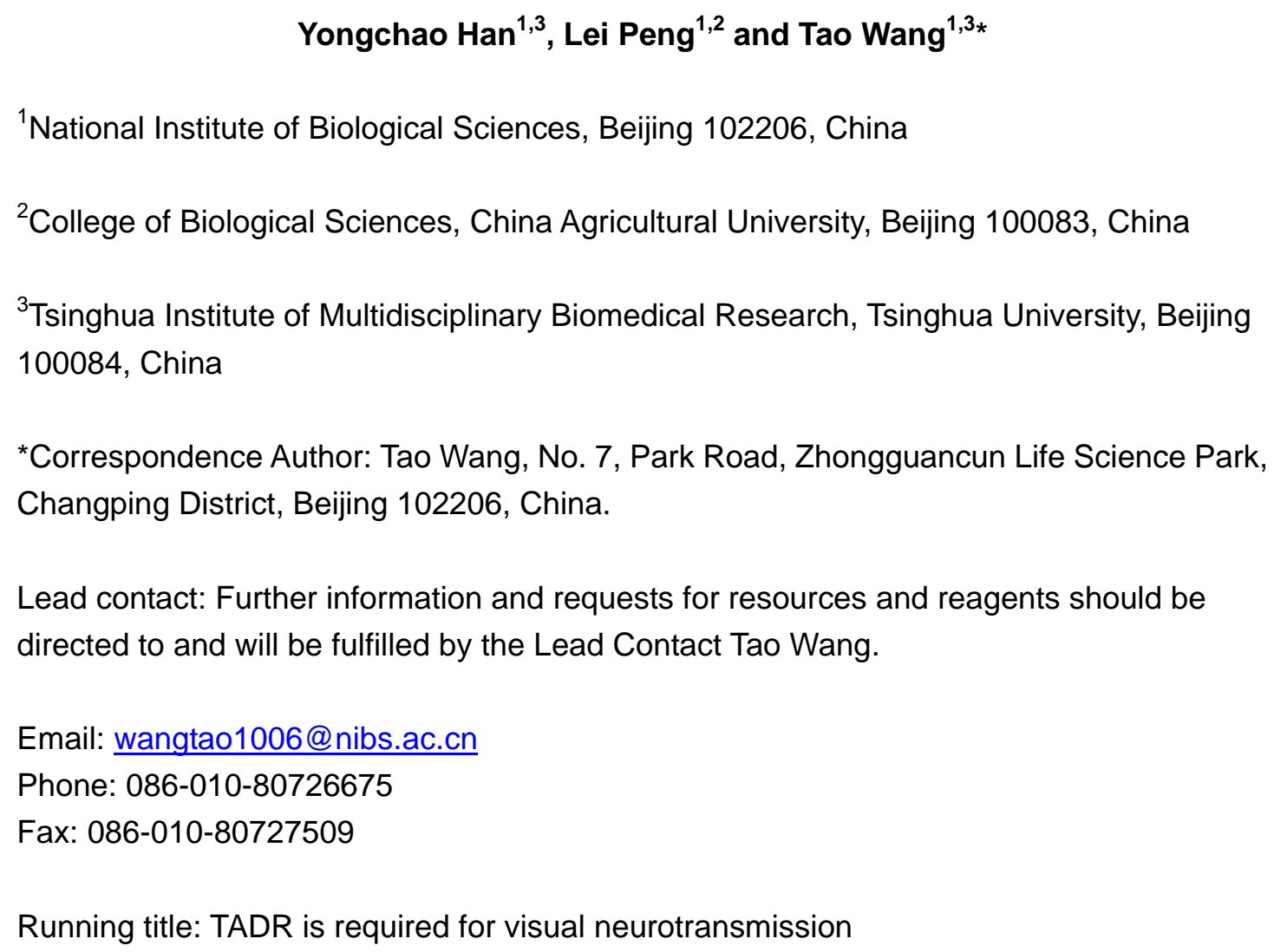


Abstract

Neurotransmitters are generated by de novo synthesis and are essential for sustained, high-frequency synaptic transmission. Histamine, a monoamine neurotransmitter, is synthesized through decarboxylation of histidine by Histidine decarboxylase (Hdc). However, little is known about how histidine is presented to Hdc as a precursor. Here, we identified a specific histidine transporter, TADR (Torn And Diminished Rhabdomeres), which is required for visual transmission in Drosophila. Both TADR and Hdc localized to neuronal terminals, and mutations in tadr reduced levels of histamine, thus disrupting visual synaptic transmission and phototaxis behavior. These results demonstrate that a specific amino acid transporter provides precursors for monoamine neurotransmitters, providing the first genetic evidence that a histidine amino acid transporter plays a critical role in synaptic transmission. These results suggest that TADR-dependent local de novo synthesis of histamine is required for synaptic transmission.

\section{Introduction}

Monoamine neurotransmitters including dopamine, serotonin, and histamine are formed primarily by the decarboxylation of amino acids (McKinney et al., 2001; Watanabe et al., 1984). Deficiencies in the biosynthesis of monoamine neurotransmitter such as dopamine contribute to a range of neurological disorders, such as dystonic and parkinsonian syndromes (Kurian et al., 2011). It has been proposed that precursor amino acids are taken up into synaptic terminals by specific transporters, followed by the synthesis and packaging of neurotransmitters within the nerve endings (Bellipanni et al., 2002; Hansson et al., 1999; Lebrand et al., 1996). However, to date, amino acid transporters specific for the synthesis of monoamine neurotransmitters have not been identified. Moreover, biosynthetic enzymes involved in the synthesis of neurotransmitters localize to both the soma and axonal terminals. Thus, it is possible that neurotransmitters are made in the cell body of presynaptic cells and then packed into synaptic vesicles and transported to axonal terminals via fast axonal transport (Broix et al., 2021; Roy, 2020).

Histamine was first identified as a neurotransmitter that localized to the tuberomamillary nucleus where it was synthesized from the amino acid histidine through a reaction catalyzed by the enzyme histidine decarboxylase (HDC), which removes a carboxyl group from histidine (Taguchi et al., 1984; Watanabe et al., 1984). As a neurotransmitter, histamine plays important roles in regulating multiple physiological processes, including cognition, sleep, synaptic plasticity, and feeding behaviors (Bekkers, 1993; Haas et al., 2008; Huang et al., 2001; Parmentier et al., 2002; Vorobjev et al., 1993). Disruption of histaminergic neurotransmission is associated with a range of neurological disorders, includding schizophrenia and multiple neurodegenerative diseases (Haas and Panula, 2003; Klaips et al., 2018; Lim and Yue, 2015; Olivero et al., 2018; Panula and Nuutinen, 2013; Wang et al., 2017). Moreover, loss of function mutations in the HDC gene lead to Tourette syndrome, a neurological disorder characterized by sudden, repetitive, rapid, and unwanted movements in both human patients and mouse models (Baldan et al., 2014; Ercan-Sencicek et al., 2010). However, a specific histidine transporter that maintains the histidine pool and delivers histidine to synaptic Hdc for histamine synthesis has not been identified. 
Drosophila photoreceptor cells use histamine as the dominant neurotransmitter to convey visual signals. Thus, generation of high levels of histamine in photoreceptor neuronal terminals is important for rapid and high-frequency visual signaling (Borycz et al., 2002; Hardie, 1989; Stuart, 1999; Wang and Montell, 2007). Similar mechanisms of histamine synthesis, storage, and release between mammals and flies make the fly a powerful molecular-genetic system for studying the metabolism of neuronal histamine (Burg et al., 1993; Chaturvedi et al., 2014; Deshpande et al., 2020; Gengs et al., 2002; Gisselmann et al., 2002; Hardie, 1989; Martin and Krantz, 2014; Wyant et al., 2017; Xu and Wang, 2019). Histamine signals are enriched in photoreceptor terminals, and disrupting histamine synthesis by $\mathrm{Hdc}$ mutation results in reduced levels of axonal histamine and loss of visual transduction. This indicates that histamine is synthesized directly within photoreceptor terminals (Chaturvedi et al., 2014; Melzig et al., 1996). In support of this notion, LOVIT, a new vesicular transporter required for the concentration of histamine in photoreceptor terminals, is exclusively found in synaptic vesicles at photoreceptor terminals (Xu and Wang, 2019).

Given the high demand for histamine to maintain visual transmission at high frequencies, we hypothesized that a histidine-specific transporter must localize to neuronal terminals, and that this transporter would be required for de novo synthesis of histamine through Hdc. In support of this hypothesis, we found that Hdc localized exclusively to photoreceptor terminals. We performed a targeted RNAi screen for transporters involved in visual transmission and identified TADR (Torn And Diminished Rhabdomeres), a plasma membrane transporter capable of transporting histidine into cells. TADR localized predominantly to photoreceptor terminals and specifically transported the amino acid histidine. Mutations in the tadr gene disrupted photoreceptor synaptic transmission, phototaxis behaviors, and levels of axonal histamine in photoreceptors. We therefore propose that a specific amino acid transporter provides precursors for the synthesis of monoamine neurotransmitters. We further provide evidence that neurotransmitters can be synthesized de novo in a specific location. 


\section{Results}

\section{Histidine decarboxylase (Hdc) localizes to neuronal terminals}

Histamine acts as major neurotransmitter at photoreceptor synaptic terminals, transmitting visual information to interneurons (Hardie, 1989). Further, histamine de novo synthesis in photoreceptor cells is essential for maintaining visual transmission (Burg et al., 1993). Interestingly, we have identified a vesicle transporter specific for histamine, LOVIT, which is concentrated exclusively in photoreceptor terminals and helps to maintain levels of histamine at synapses (Xu and Wang, 2019). Together with the fact that visual neurotransmission requires rapid and high-frequency firing, we hypothesize that the fast neurotransmitter histamine is synthesized directly in axon terminals. In Drosophila photoreceptor cells, histamine is initially synthesized from histidine by the eye-specific enzyme, histidine decarboxylase ( $\mathrm{Hdc}$ ). To determine the subcellular localization of Hdc in photoreceptor cells, we raised an antibody against an Hdc-specific peptide. Endogenous Hdc was detected predominantly in the lamina layer, which contains terminals of the R1-R6 photoreceptors, and in the medulla, which contains terminals of R7- R8. In these regions, Hdc co-localized with LOVIT and the pre-synaptic marker, CSP (Cysteine String Protein) (Figures 1A-B, and Figure 1 -figure supplement 1 ). Moreover, Hdc was largely absent from the retina (Figures $1 \mathrm{~A}$ and $1 \mathrm{~B}$ ). Cross-sections of the lamina neuropil that contained R1-R6 terminals revealed that Hdc was surrounded by the glial marker Ebony and co-localized with synaptic vesicle protein CSP (Figures 1C). To confirm the sub-cellular pattern of Hdc, we expressed mCherry-tagged Hdc in photoreceptor cells using the trp promoter (transient receptor potential) (Montell and Rubin, 1989). The chimeric Hdc was functional, as the trp-Hdc-mCherry transgene completely restored visual transmission in $\mathrm{Hdc}$ mutant flies. Consistent with what we observed for endogenous Hdc, Hdc-mCherry was also highly enriched in the lamina and medulla. In contrast, GFP signals were detected in the retina, lamina, and medulla in trp-GFP flies (Figure 1D). The finding that Hdc protein was enriched in photoreceptor terminals is consistent with the assumption that the neurotransmitter histamine is synthesized directly in axon terminals.

\section{TADR is required for visual synaptic transmission}

Given that the enzyme responsible for catalyzing the biosynthesis of histamine localized to pre-synaptic regions, we next sought to determine how histidine, an Hdc substrate, is transported to neuronal terminals. We hypothesized that an amino acid transporter resided on the plasma membrane of photoreceptor synaptic terminals, and that this transporter would be responsible for histidine uptake and required for rapid histamine synthesis and visual transmission. Among $\sim 600$ putative transmembrane transporters encoded by the Drosophila genome (Ren et al., 2007), we identified 42 genes encoding SLC1, SLC6, SLC7, SLC17, SLC25, SLC32, SLC36, SLC38, and SLC66 families of proteins that were predicted to import amino acids across the plasma membrane (Bröer and Bröer, 2017). These we tested as candidate histidine transporters (Table 1).

To examine whether these putative transporters were involved in visual neurotransmission, each candidate gene was knocked down individually via the eye-specific expression of RNAi using the GMR (glass multiple response element)-Gal4 driver. Loss-of-function alleles were 
also used if available. We performed electroretinogram (ERG) recordings to determine which putative amino acid transporters functioned in visual transmission. ERG recordings are extracellular recordings that measure the summed responses of all retinal cells in response to light. An ERG recording from a wild-type fly contains a sustained corneal negative response resulting from photoreceptor depolarization, as well as ON and OFF transients originating from synaptic transmission to laminal LMCs (large monopolar cells) at the onset and cessation of light stimulation (Wang and Montell, 2007) (Figure 2A). Flies deficient for histamine exhibited clear reductions in their ON and OFF transients, as shown for $\mathrm{Hdc}^{P 217}$ mutant flies (Burg et al., 1993; Melzig et al., 1996). We found that knock-down of the putative cationic amino acid transporter gene, tadr (torn and diminished rhabdomeres), resulted in the loss of synaptic transmission (Ni et al., 2008) (Figures 2A-2B and Figure 2-figure supplement 1). We then generated a different $\operatorname{tadr}^{R N A i}$ line, which we called $\operatorname{tadr}^{\text {RNAi-2 }}$. Consistent with the results of the screen, driving $\operatorname{tadr}^{\text {RNAi-2 }}$ with GMR-gal4 also affected ON and OFF transients (Figures $2 \mathrm{~A}$ and 2B). Importantly, ERG transients were not affected by specific knockdown of tadr in glia using repo-Gal4, confirming the specific role of TADR in photoreceptor neurons (Figures 2C and 2D).

To further confirm that tadr was the causal gene, we generated a null mutation in the tadr gene by deleting a 665-bp genomic fragment using the CRISPR-associated single-guide RNA system (Cas9) (Figure 2-figure supplement 2A). PCR amplification and sequencing of the tadr locus from genomic DNA isolated from wild-type and $\operatorname{tadr}^{2}$ flies revealed a truncated tadr locus in mutant animals, resulting in an out-of-frame fusion of exons 3 and 5 (Figure 2-figure supplement $2 \mathrm{~B}$ and $2 \mathrm{C}$ ). It has been reported that tadr mutation leads to photoreceptor degeneration. However, homozygous tadr ${ }^{2}$ null mutants exhibited normal morphology of both the soma and axon terminal of photoreceptors when examined via transmission electron microscopy (TEM) (Figure 2-figure supplement 3). Furthermore, no retinal degeneration was observed in aged tadr $^{2}$ mutant flies. As a control, aged culd ${ }^{1}$ mutants exhibited extensive rhobdomere loss, which resulted from disruption of rhodopsin endocytic trafficking ( $\mathrm{Xu}$ and Wang, 2016) (Figure 2-figure supplement 3A and 3B). Consistent with the RNAi results, tadr ${ }^{2}$ mutant flies displayed a complete loss of ON and OFF transients (Figure 2E). Further, expressing tadr in photoreceptors using the trp promoter restored ON and OFF transients in $\operatorname{tadr}^{2}$ mutant flies, whereas expression of GFP failed to rescue the loss of ERG transients (Figures 2E and 2F). Disrupting visual transmission results in blindness, which reflected in the loss of phototactic behavior (Behnia and Desplan, 2015). We next used this behavioral assay to assess the vision of tadr mutant flies. Consistent with the ERG results, knockdown of tadr in the retina disrupted phototactic behavior, whereas wild-type levels of phototaxis were observed in flies in which tadr was knocked down in glia (Figure 2G). $\operatorname{tadr}^{2}$ mutant flies also exhibited defective phototaxis, which was fully restored by the trp-tadr transgene (Figure 2H). Together, these findings reveal that TADR functions within photoreceptor cells to maintain synaptic transmission but not the integrity of neurons.

\section{TADR is a bona fide histidine transporter}

Given that TADR belongs to a subfamily of cationic amino acid transporters (CATs) within the solute carrier 7 (SLC7) family (Verrey et al., 2004), we performed a histidine uptake assay in Drosophila S2 cells to determine whether TADR could transport histidine in vitro (Han et al., 
2017; Karl et al., 1989). When Flag-tagged TADR was expressed in S2 cells the Flag-TADR signal localized exclusively to the plasma membrane (Figure $3 \mathrm{~A}$ ). We then transiently expressed TADR in S2 cells and assessed their ability to uptake $\left[{ }^{3} \mathrm{H}\right]$-histidine. The histidine content of TADR-transfected cells was approximately $180 \mathrm{~Bq} / \mathrm{mg}$, which was 3.6-fold greater than measured for RFP-transfected controls ( $50 \mathrm{~Bq} / \mathrm{mg}$ ) (Figure 3B). Human SLC38A3, which is known to efficiently take up histidine, exhibited levels of histidine transport comparable to TADR, suggesting that TADR is a bona fide plasma membrane histidine transporter (Bröer, 2014) (Figure 3B).

Considering that transporters related to histamine recycling are necessary for synaptic transmission, we next sought to determine whether TADR specifically transports histidine in Drosophila. Because a histamine transporter has not yet been identified, we first asked whether TADR can transport histamine. Histamine uptake assays revealed that TADR does not exhibit histamine uptake activity. As a control, the human Organic Cation Transporter (OCT2), which is known to take up histamine, exhibited high levels of histamine transport when expressed in S2 cells (Busch et al., 1998) (Figure 3C). Next, we found that TADR did not exhibit $\beta$-alanine transporting activity when expressed in S2 cells, whereas BalaT, which served as a positive control, efficiently transported $\beta$-alanine (Han et al., 2017) (Figure 3D). Similarly, TADR did not transport carcinine in S2 cells compared with the positive control, OCT2 (Xu et al., 2015) (Figure 3E). To further determine if the amino acid transporter TADR is specific to histidine, we performed competition assays using $\left[{ }^{3} \mathrm{H}\right]$-histidine in combination with high concentrations of different L-amino acids or carcinine $(0.5 \mathrm{mM}$ for each L-amino acid or carcinine vs. $2.5 \mu \mathrm{M}\left[{ }^{3} \mathrm{H}\right]$-histidine). Histidine efficiently blocked $\left[{ }^{3} \mathrm{H}\right]$-histidine uptake, whereas the other amino acids and carcinine did not affect TADR-mediated histidine uptake. The only exception was lysine, which slightly reduced $\left[{ }^{3} \mathrm{H}\right]$-histidine uptake (Figure 3-figure supplement 1). These data support the conclusion that TADR is a specific histidine transporter involved in visual synaptic transmission.

\section{TADR predominantly localizes to photoreceptor terminals}

Hdc and LOVIT mediate two steps critical for histamine synthesis and we found that both localize to axonal terminals. Thus, if TADR functions as a histidine transporter epistatic to Hdc, we should also detect TADR in photoreceptor axonal terminals. As we failed to generate a high affinity antibody against TADR, we used CRISPR/Cas9-based genome editing to introduce a GFP tag into the tadr locus (tagging the N-terminal), downstream of the native tadr promoter (GFP-tadr) (Figure 4-figure supplement $1 \mathrm{~A}$ and method). The reason for generating an $\mathrm{N}$-terminal tagged version of TADR is that expression of $\mathrm{N}$-terminal tagged but not $\mathrm{C}$-terminal tagged TADR fully restored synaptic transmission in $\operatorname{tadr}^{\text {RNAi-2 }}$ flies. We identified GFP-tadr knock-in flies though PCR (Figure 4-figure supplement 1B). Importantly, homozygous GFP-tadr flies displayed intact ON and OFF transients, as expected, confirming that GFP-TADR retained in vivo function (Figure 4-figure supplement $1 \mathrm{C}$ ). We found that TADR protein was enriched in photoreceptor cells including the retina, lamina, and medulla. Importantly, the GFP-TADR signal was concentrated in the lamina and medulla (marked with $\mathrm{CSP}$ ), to which the R1-R6 and R7/R8 photoreceptors project their axons (Figure 4A). Moreover, both TADR and Hdc localized to terminals of photoreceptor neurons in the lamina 
and medulla (Figure 4B). These results demonstrated that TADR is expressed specifically in photoreceptor neurons and localized primarily to photoreceptor terminals. Further, the pattern of Hdc localization suggests that the de novo synthesis of a neurotransmitter occurs specifically at the relevant synapse.

\section{tadr mutants exhibit reduced levels of histamine at photoreceptor terminals}

Given our evidence consistent with TADR-mediated transport of histidine directly into photoreceptor synaptic terminals, where histidine would be converted to histamine by Hdc, loss of TADR should reduce histamine levels, as has been seen for hdc mutants (Borycz et al., 2000). We generated head longitudinal sections from $\operatorname{tadr}^{2}$ mutants and wild-type controls and labeled them with an antibody reported to label histamine (Chaturvedi et al., 2014). In control flies, the histamine signal was enriched exclusively in photoreceptor terminals of both the lamina and medulla, co-localizing with CSP (Figure 5A and 5C). tadr ${ }^{2}$ mutants showed a dramatic reduction in histamine labeling at photoreceptor terminals in both the lamina and medulla (Figure 5B and 5C). Moreover, the apparent loss of histamine in $\operatorname{tadr}^{2}$ mutant photoreceptor terminals did not result from the loss of synaptic structures, as the density of synaptic vesicles and the number of capitate projections were comparable in the lamina of tadr $r^{2}$ mutant and control flies (Figure 2-figure supplement 3). We next used liquid chromatography-mass spectrometry (LC-MS) to examine in vivo levels of histamine in compound eyes of $\operatorname{tadr}^{2}$ mutant flies. As expected, eyes from $\mathrm{Hdc}^{P 217}$ mutant flies exhibited reduced levels of histamine, as these flies could not decarboxylate histidine into histamine (Figure 5D). Similarly, $\operatorname{tadr}^{2}$ mutants produced less histamine due to impairment of histidine uptake into photoreceptor terminals (Figure 5D). Moreover, histamine levels in $\operatorname{tadr}^{2}$ mutant flies were higher than in $\mathrm{Hdc}^{P 217}$ mutant flies, suggesting that a small fraction of histidine could be supplied by other transporter systems. Consistent with previous reports, examining the heads of $H d c^{P 217}$ mutant flies revealed less carcinine and $\beta$-alanine due to loss of histamine (Melzig et al., 1998) (Figures 5E and 5F). Importantly, tadr $^{2}$ mutants exhibited less carcinine and $\beta$-alanine as well, indicating that both TADR and Hdc are essential for the de novo synthesis of histamine. Moreover, reductions in histamine, carcinine, and $\beta$-alanine in $t a d r^{2}$ mutants were fully restored by expressing TADR in photoreceptor cells (Figures $5 E$ and $5 F$ ). Reduction of histamine in $\operatorname{tadr}^{2}$ mutant flies indicates defective histamine synthesis and explains why photoreceptor synaptic transmission is disrupted in $t a d r^{2}$ mutants. These results therefore support the hypothesis that TADR transports histidine into photoreceptor terminals for the production of histamine to sustain tonic visual transmission.

\section{Ectopic expression of histindine transporters in photoreceptor cells rescues visual synaptic transmission in $\operatorname{tadr}^{2}$ flies.}

If tadr mutants exhibit defective visual transmission because of deficient histidine transport, then replacing tadr with another transporter capable of transporting histidine should rescue photoreceptor synaptic transmission in tadr mutants. We first asked whether other members of the fly CAT family of transporters could efficiently transport histidine into S2 cell. We found that histidine was taken up by CG13248-transfected cells, but not by Slif (Slimfast)-positive cells (Figure 6A). Next, we overexpressed CG13248 or Slif in $\operatorname{tadr}^{2}$ mutant photoreceptor cells. The 
259 expression of CG13248 in $\operatorname{tadr}^{2}$ mutant photoreceptor cells fully restored both ERG transients

260 and phototaxis, whereas Slif did not. These results are consistent with their abilities to

261 transport histidine (Figures 6B-6D). Further, expression of the human histidine transporter,

262 SLC38A3, fully restored ERG transients and phototactic behavior in $t a d r^{2}$ mutant flies (Figures

$2636 \mathrm{~A}-6 \mathrm{D}$ ). These data support an essential role for the histidine transporting activity of TADR in

264 maintaining visual synaptic transmission. Taken together, we have identified a previously

265 uncharacterized histidine transporter, TADR, and shown that TADR resides (together with the

266 downstream enzyme, $\mathrm{Hdc}$ ) in the axonal terminals of photoreceptor neurons, where it is responsible for the local biosynthesis of histamine.

268 


\section{Discussion}

270 Neurotransmitters are concentrated within presynaptic terminals and their release transmits signals to postsynaptic neurons. In most cases, the enzymes necessary for neurotransmitter synthesis are translated in the soma and then transported down the axon, where they then generate neurotransmitters. However, Tyrosine hydroxylase and Tryptophan hydroxylase, the rate-limited enzymes for dopamine and serotonin synthesis, respectively, are cytosolic and reside both in the neuronal cell body and axon, suggesting that the de novo synthesis of these neurotransmitters occurs in both the soma and axon (Cartier et al., 2010; Walther et al., 2003). If neurotransmitters are generated in the cytosol of the cell body, the slow rate of diffusion for these small molecules could potentially limit the pool of axonal neurotransmitters and affect synaptic transmission. One possibility is that neurotransmitters are loaded into storage vesicles and that these vesicles are then taken to the nerve endings through fast axonal transport (Broix et al., 2021; Roy, 2020; Vallee and Bloom, 1991). However, the recently identified vesicular transporter, LOVIT, which is responsible for packaging histamine, is absolutely restricted to synaptic vesicles within the photoreceptor axon. Histamine is exclusively detected in wild-type photoreceptor terminals, but absent from lovit mutants (Xu and Wang, 2019). Consistent with this, we found that Hdc and its product histamine are enriched in photoreceptor terminals, suggesting that the de novo synthesis of histamine occurs exclusively in axons. If histidine, the substrate of $\mathrm{Hdc}$, is transported to axonal terminals from the cell body, the rate of histamine biosynthesis would be limited. Therefore, it is possible that neurotransmitter precursors are taken up into terminals by specific transporters, and that neurotransmitter synthesis and packaging take place primarily within the axon. In support of this, we have characterized a new histidine transporter, TADR, which localized predominantly to photoreceptor terminals and was required for the de novo synthesis of histamine in photoreceptor terminals. Furthermore, TADR specifically transported histidine in vitro, and tadr $^{2}$ null mutant flies exhibited normal neuronal growth and survival, but disrupted visual transmission. Therefore, TADR and Hdc function synergistically within axonal terminals to provide a local pool of neurotransmitters (Figure 6E).

Although de novo synthesis provides a starting pool of neurotransmitters, recycling neurotransmitters after release is a critical pathway for maintaining neurotransmitter content within axon terminals. Ebony, the histamine recycling pathway involved in a previously identified $\mathrm{N}$ - $\beta$-alanyl-dopamine synthase, is expressed in epithelial glia and converts histamine to carcinine. The inactive histamine conjugate, carcinine, is transported back into photoreceptors where it is hydrolyzed back into histamine by Tan, an N- $\beta$-alanyl-dopamine hydrolase, to restore the neurotransmitter pool (Borycz et al., 2002; Richardt et al., 2003; Richardt et al., 2002; Wagner et al., 2007). Unlike Hdc, Tan localizes non-selectively to both the soma and axon, suggesting that the regeneration of histamine may take place in the soma as well (Aust et al., 2010). However, a recently identified transporter specific for carcinine, CarT, predominantly localizes to the terminals of photoreceptor neurons, rather than to the cell bodies, suggesting the regeneration of histamine from carcinine through axonal Tan (Chaturvedi et al., 2016; Stenesen et al., 2015; Xu et al., 2015). Taken together, both de novo 
synthesis and regeneration of the neurotransmitter histamine is restricted to neuronal terminals by a similar mechanism - the transportation of substrates.

TADR belongs to the CAT subfamily within the SLC7 family, and is homologous to the human membrane proteins, SLC7A4 and SLC7A1, which are predicted to be involved in importing basic amino acids across the plasma membrane (Verrey et al., 2004). It has been reported that flies carrying a missense mutation in tadr, namely $\operatorname{tadr}^{1}$ (generated from an EMS mutagenesis screen), exhibit retinal degeneration. This may indicate that TADR functions to provide amino acids to support photoreceptor cells (Ni et al., 2008). However, our tadr ${ }^{2}$ null mutant flies fail to exhibit photoreceptor degeneration or growth defects. In addition, the morphologies of tadr ${ }^{2}$ somas and axons are comparable to those of wild-type flies. In support of this, we found that TADR specifically transported histidine, with only a low affinity for lysine, suggesting that TADR is not involved in the general metabolism of amino acids. The neurodegeneration phenotype observed in $\operatorname{tadr}^{1}$ mutants may be due to other mutations generated by EMS mutagenesis or the neomorphic tadr $^{1}$ point mutation, which may disrupt $\mathrm{Gq}$ signaling (Ni et al., 2008). The CG13248 transporter, a member of the CAT subfamily, is able to rescue the vision defects of tadr ${ }^{2}$ mutant flies, but another member of the CAT subfamily, the transporter Slif, failed to do so. This is consistent with their ability to transport histidine (Colombani et al., 2003). Moreover, SLC38A3, which belongs to the SLC38 subfamily, is known to mediate sodium-dependent transport of multiple amino acids, including histidine, and the expression of SLC38A3 fully restores visual transduction and phototactic behavior in tadr $^{2}$ mutant flies (Bröer, 2014). The lack of sequence homology between SLC38A3 and TADR strongly suggests that TADR primarily acts as a histidine transporter to maintain visual transduction. Although TADR is a specific histidine transporter, its expression patterns suggests that it is not the only histidine transporter. Supporting this, null tadr mutants are viable and exhibit no growth or cell death phenotypes. If photoreceptor cells uptake histidine via other histidine transporters, they could have a small histidine pool and Hdc would be able to synthesize some histamine. In agreement with this, histamine levels in $\operatorname{tadr}^{2}$ mutant are higher than in $\mathrm{Hdc}$ mutant, although histamine levels in both mutants are greatly reduced. Consistent with these relative histamine levels, $\operatorname{tadr}^{2}$ mutation exibit more phototactic behavior than Hdc mutants.

Amino acid transporters play fundamental roles in multiple metabolic processes, including mTOR activation, energy metabolism, nutritional stress, and tumor progression (Chen et al., 2014; Colombani et al., 2003; Nicklin et al., 2009; Rebsamen et al., 2015; Wyant et al., 2017). Consistent with these functions, the amino acid transporters SLC7A5 and SLC6A14 are upregulated in tumors, making then potential targets for the pharmacological treatment of cancer (Kanai, 2021; Nałęcz, 2020). Our experiments suggest that amino acid transporters provide amino acids that are critical for the de novo synthesis of monoamine neurotransmitters. Since, inhibitors of monoamine transporters have been widely used as antidepressants, amino acid transporters specific for monoamine neurotransmitter synthesis (such as TADR) may provide new treatment options for neurological diseases associated with the dysregulation of monoamine neurotransmitters (Andersen et al., 2009). 


\section{Figure legends}

Figure 1. Histidine decarboxylase (Hdc) localizes to neuronal terminals.

(A-B) Cryosections of $w^{1118}$ and $\mathrm{Hdc}$ mutant heads were labeled with antibodies against $\mathrm{Hdc}$ (red), 24B10 (A) (green, photoreceptor cell marker), CSP (B) (green, localized to synaptic vesicles), and LOVIT (blue, labelling photoreceptor terminals). Scale bars, $50 \mu \mathrm{m}$. (C) Cross sections of the lamina layer showing overlapping patterns of Hdc (red) and CSP (green) localization, and a complementary pattern of $\mathrm{Hdc}$ (red) and Ebony (blue, expressed in lamina epithelial glia). Scale bars, $5 \mu \mathrm{m}$. (D) Cryosections of heads from trp-Hdc-mCherry/trp-GFP flies were labeled with antibodies against mCherry (red) and GFP (green). Scale bar, $50 \mu \mathrm{m}$. La, lamina; Me, medulla; Re, retina.

Figure 2. TADR is required in photoreceptors for normal visual transmission.

(A-D) ERGs recorded from flies expressing various UAS-tadr RNAi transgenes (tadr ${ }^{\text {RNAit }}$ and $\operatorname{tadr}^{\text {RNAi2}}$ ) under the control of (A) GMR-Gal4, a driver specific for compound eyes (GMR-Gal4/ UAS-tadr ${ }^{R N A i 1}$ or GMR-Gal4/+;UAS-tadr ${ }^{R N A i 2} /+$ ) and (C) the glial-specific driver repo-Gal4 (repo-Gal4/UAS-tadr ${ }^{R N A i 1}$ or repo-Gal4/+;UAS-tadr $r^{R N A i 2} /+$ ). (B) Quantitative analysis of the amplitudes of ERG OFF transients shown in A compared with control flies (GMR>GFP ${ }^{R N A i}$, GMR-Gal4/+;UAS-GFP $P^{R N A i} /+$ ) (One-way ANOVA; $n=5 ;{ }^{* * *} \mathrm{p}<0.001$ ). (D) Quantitative analysis of the amplitudes of ERG OFF transients shown in $C$ compared with control flies (repo>GFP ${ }^{R N A i}$, repo-Gal4/UAS-GFP ${ }^{R N A i}$ ) (One-way ANOVA; n=10; ns, not significant). Arrowheads indicate ON and OFF transients. One-day-old flies were dark adapted for $1 \mathrm{~min}$ and subsequently exposed to a 5-s pulse of orange light. (E-F) ERG recordings (E) and quantitative analysis of the amplitudes of ERG OFF transients $(F)$ from wild-type $\left(w^{1118}\right)$, tadr $r^{2}$, $t a d r^{2}$;trp-GFP, and tadr'; ;rp-tadr flies. Displayed are comparisons to wild-type $\left(w^{1118}\right)$ flies (One-way ANOVA; $n=10 ;{ }^{* * *} p<0.001$; ns, not significant). (G) Phototactic behavior of flies corresponding to those in $(\mathrm{A})$ and $(\mathrm{C})$ compared with control flies (GMR>GFP $P^{\text {RNAi }}$ or repo->GFP ${ }^{R N A i}$ flies). (H) Phototactic behavior of flies corresponding to those in (E). Each group is comprised of at least 203 -day-old flies. Five repeats were quantified for each group (One-way ANOVA, ${ }^{* * *} p<0.001 ; n s$, not significant).

\section{Figure 3. TADR is a plasma membrane histidine transporter.}

(A) TADR localized to the plasma membrane when transiently transfected into S2 cells. GFP-tagged TADR was labeled with GFP antibody (green) and DAPI (blue), which stained the nucleus. Scale bar, $2 \mu \mathrm{m}$. (B) TADR transported histidine into S2 cells. Human SLC38A3 and RFP were used as positive and negative controls, respectively. $\left[{ }^{3} \mathrm{H}\right]$-histidine was added to the DMEM solution (final concentration $2.5 \mu \mathrm{M}$ ). (C-D) TADR did not transport histamine (C) or $\beta$-alanine (D) into S2 cells. $\left[{ }^{3} \mathrm{H}\right]$-histamine or $\left[{ }^{3} \mathrm{H}\right]$ - $\beta$-alanine was added to the ECF buffer (final concentration $3.7 \times 10^{4} \mathrm{~Bq}$ ), and OCT2 and BalaT served as positive transporter controls for histamine and $\beta$-alanine, respectively. Results are the mean \pm S.D. of three experiments (One-way ANOVA, ${ }^{* * *} \mathrm{p}<0.001$; ns, not significant). (E) TADR did not transport carcinine. S2 cells transiently expressing mCherry, OCT2-mCherry, or TADR/mCherry. Carcinine was added 
to the culture medium at a final concentration of $20 \mu \mathrm{M}$. Cells were labeled with rabbit anti-carcinine (green) antibody and DAPI (blue). The mCherry (red) signal was observed directly. Scale bar, $5 \mu \mathrm{m}$.

Figure 4. TADR predominantly localizes to photoreceptor terminals.

(A-B) Cryosections from GFP-tadr knock-in flies. Expression of an N-terminal GFP-tagged version of TADR was driven by the native tadr promoter. Sections were labeled for GFP-TADR with CSP (red) (A) or Hdc (red) (B) and DAPI (blue). Scale bars, $50 \mu \mathrm{m}$. La, lamina; Me, medulla; Re, retina.

\section{Figure 5. Loss of TADR reduces histamine levels in vivo.}

(A-C) Histamine signaling in photoreceptor terminals was disrupted. Head cryosections were stained for histamine together with CSP (synaptic vesicle marker) in control $\left(w^{1118}\right)(A)$ and $\operatorname{tadr}^{2}$ (B) flies. Sections are parallel to photoreceptor axons. Scale bars, $50 \mu \mathrm{m}$. (C) Average red fluorescence intensity ratio between the entire lamina and the retina immunolabeled layers. (D-F) Histamine (D), $\beta$-alanine (E), and carcinine (F) levels in compound eyes of 3-day-old control $\left(w^{1118}\right), H d c^{P 217}, t a d r^{2}$, and $\operatorname{tadr}^{2}$; trp-tadr flies. Each sample included dissected compound eyes from 40 flies (One-way ANOVA; $n=4 ;{ }^{* * *} p<0.001 ;{ }^{* *} p<0.01 ;{ }^{*} p<0.05$ ). La, lamina; Me, medulla; Re, retina.

Figure 6. Rescue of defective visual transmission in $\operatorname{tadr}^{2}$ mutants by expressing other histidine transporters.

(A) SLC38A3 and CG13248 transported histidine into S2 cells, whereas the previously identified amino acid transporter, Slif, did not transport histidine. $\left[{ }^{3} \mathrm{H}\right]$-histidine was added to the DMEM solution to a final concentration $2.5 \mu \mathrm{M}$. (B) ERG recordings from control $\left(w^{1118}\right)$, tadr $r^{2}, \operatorname{tad}^{2}+\operatorname{tad} r\left(\operatorname{tadr}^{2} ;\right.$;long GMR-Gal4/UAS-tadr), tadr $r^{2}+C G 13248$ (tadr ${ }^{2}$;longGMR-Gal4/UAS-CG13248), tadr $^{2}+$ Slif (tadr ${ }^{2}$;longGMR-Gal4/UAS-Slif), and tadr $^{2}+$ SLC38A3 (tadr ${ }^{2} ;$ longGMR-Gal4/UAS-SLC38A3) are shown. Young flies ( $<3$ days after eclosion) were dark adapted for $1 \mathrm{~min}$ and subsequently exposed to a 5-s pulse of orange light. (C) Quantitative analysis of the amplitude of ERG OFF transients shown in B. Displayed are comparisons to control $\left(w^{1118}\right)$ flies (One-way ANOVA; $n=10$; ${ }^{* \star *} \mathrm{p}<0.001$; ns, not significant). (D) Phototactic behaviors of 3-day-old control, $\operatorname{tadr}^{2}, \operatorname{tadr}^{2}+\operatorname{tad} r, \operatorname{tadr}^{2}+C G 13248, \operatorname{tad}^{2}+$ Slif, and $t a d r^{2}+S L C 38 A 3$ flies. Five repeats were made for each group, and each group had at least 20 flies (One-way ANOVA, ${ }^{\star \star *} \mathrm{p}<0.001$; ns, not significant). (E) Model of the pathway for local histamine biosynthesis. Histidine is directly transported into photoreceptor cells at neuronal terminals by TADR, where it is used as a substrate to synthesize histamine by the decarboxylase, Hdc. Newly generated histamine is then loaded into synaptic vesicles by a LOVIT-dependent mechanism. Histamine, serving as a neurotransmitter, is released by photoreceptor cells $(\mathrm{PR})$ to activate histamine-gated chloride channels (HisClA) on postsynaptic neurons (LMC) to start visual transmission.

Figure 1-figure supplement 1. Histidine decarboxylase (Hdc) co-localizes with LOVIT in the medulla. 
429 A longitudinal section of the distal medulla neuropil from 3-day-old $w^{1118}$ fly was labeled with

430 antibodies against Hdc (red) and LOVIT (blue, labelling photoreceptor terminals). Scale bars,

$43120 \mu \mathrm{m}$.

Figure 2-figure supplement 1. ERG recordings for putative amino acid transporters functioned in visual transmission.

ERGs recorded from flies knocking down 42 putative amino acid transporter genes under GMR-Gal4. One-day-old flies were dark adapted for $1 \mathrm{~min}$ and subsequently exposed to a 5-s pulse of orange light.

Figure 2-figure supplement 2. Generation of $\operatorname{tadr}^{2}$ flies.

(A) Schematic for tadr knock-out by sgRNA targeting. Organization of the tadr locus and the expected structure of the $\operatorname{tadr}^{2}$ allele are shown. Boxes represent exons with the coding region between ATG and TAA. The sgRNA1 and sgRNA2 primer pairs were used to generate the tadr $^{2}$ allele. Arrows indicate the primers used for genomic PCR. (B) PCR products obtained from $\operatorname{tadr}^{2}$ mutants show successful gene deletions of $\sim 600 \mathrm{bp}$. (C) Verification of the $t a d r^{2}$ locus by DNA sequencing. The $t a d r^{2}$ mutation elimnates 665 bp within the tadr locus including all of exon 4 and parts of exons 3 and 5 . This leads to a deletion fo 244 nt in the spliced tadr ${ }^{2}$ mRNA, resulting in a frame shift at the truncated site, as shown.

Figure 2-figure supplement 3. Ommatidia and cartridges are normal in $\operatorname{tadr}^{2}$ mutants.

(A) Cross-view of TEM sections of the retinal layers from wild type $\left(w^{1118}\right), \operatorname{tadr}^{2}$, and culd $d^{1}$ flies at indicated days. Scale bars: $2 \mu \mathrm{m}$. (B) Quantification of the average number of rhabdomeres per ommatidium at indicated days fro wild type $\left(w^{1118}\right)$, $t a d r^{2}$, and culd $d^{1}$ flies. (C) Cross-view of TEM sections of lamina neuropil from 3-day-old $w^{1118}$ and $\operatorname{tadr}^{2}$ flies. (D-E) Quantification of capitate projection $(C)$ and synaptic vesicle density $(D)$ from $B$. Sections from four different flies were quantified for each genotype.

\section{Figure 3-figure supplement 1. TADR is a specific histidine transporter.}

(A) Competition assays using $\left[{ }^{3} \mathrm{H}\right]$-histidine in combination with high concentrations of different L-amino acids ( $0.5 \mathrm{mM}$ for each L-amino acid vs. $2.5 \mu \mathrm{M}\left[^{3} \mathrm{H}\right]$-histidine). (B) Competition assays using $\left[{ }^{3} \mathrm{H}\right]$-histidine in combination with a high concentration of carcinine $(0.5 \mathrm{mM}$ for carcinine vs. $2.5 \mu \mathrm{M}\left[{ }^{3} \mathrm{H}\right]$-histidine). Results are the mean \pm S.D. of three experiments (One-way ANOVA; ${ }^{* * *} \mathrm{p}<0.001 ;{ }^{* *} \mathrm{p}<0.01$; ${ }^{*} \mathrm{p}<0.05$; ns, not significant).

\section{Figure 4-figure supplement 1. Generation of GFP-tadr flies.}

(A) Schematic for generating GFP-tadr flies. GFP was inserted into the tadr locus (tagging the $\mathrm{N}$-terminal) using CRISPR/Cas9-mediated homologous recombination. PCR primers (arrows, $\mathrm{pF}$ and $\mathrm{pR}$ ) were used to verify the GFP-tadr knock-in flies. (B) Genomic PCR products from wild-type $\left(w^{1118}\right)$, attP2, and GFP-tadr flies show successful gene targeting. (C) ERG recordings from wild-type $\left(w^{1118}\right)$, nos-cas9, and GFP-tadr flies. Homozygous GFP-tadr flies showed intact ON and OFF transients. 
Figure 6-figure supplement 1. Both CG13248 and Slif do not transport histamine.

CG13248 and Slif did not transport histamine into S2 cells. [ $\left.{ }^{3} \mathrm{H}\right]$-histamine was added to the ECF buffer (final concentration $3.7 \times 10^{4} \mathrm{~Bq}$ ). OCT2 served as a positive transporter control for histamine. Results are the mean \pm S.D. of three experiments (One-way ANOVA, ${ }^{* *} p<0.001$; ns, not significant).

Table 1. Description of 42 putative amino acid transporters.

\section{Source data}

\section{Figure 2-source data 1}

Source data for quantitative of ERG transients and phototaxis behaviors.

Figure 2-figure supplement 2 source data 1

The full raw unedited gels for PCR products obtained fromc mutants.

\section{Figure 2-figure supplement 3 source data 1}

Source data for quantifying the average number of rhabdomeres per ommatidium at indicated days for wild type $\left(w^{1118}\right)$, tadr ${ }^{2}$, and culd ${ }^{1}$ flies, as well as for quantifying capitate projection and synaptic vesicle density in $t a d r^{2}$ mutants.

\section{Figure 3-source data 1}

Source data for histidine, histamine, and $\beta$-alanine uptake assay.

\section{Figure 3-figure supplement 1 source data 1}

Source data for competition assays using histidine in combination with different L-amino acid or carcinine.

\section{Figure 4-figure supplement 1 source data 1}

The full raw unedited gels for PCR products obtained from GFP-tadr flies.

\section{Figure 5-source data 1}

Source data for the levels of histamine, $\beta$-alanine, and carcinine in $w^{1118}, \mathrm{Hdc}^{P 217}$, $\operatorname{tadr}^{2}$, and tadr $r^{2}$;rp-tadr mutant fly compound eyes.

\section{Figure 6-source data 1}

Source data for histidine uptake assay and source data for quantitative of ERG transients and phototaxis behaviors.

Figure 6-figure supplement 1 source data 1 
Materials and Methods

\begin{tabular}{|c|c|c|c|c|}
\hline \multicolumn{5}{|c|}{ Key Resources Table } \\
\hline $\begin{array}{l}\text { Reagent } \\
\text { type } \\
\text { (species) } \\
\text { or } \\
\text { resource }\end{array}$ & Designation & $\begin{array}{l}\text { Source or } \\
\text { reference }\end{array}$ & Identifiers & $\begin{array}{l}\text { Additional } \\
\text { information }\end{array}$ \\
\hline $\begin{array}{l}\text { gene }(D . \\
\text { melanog } \\
\text { aste) }\end{array}$ & $\operatorname{tadr}(\mathrm{cDNA})$ & $\begin{array}{l}\text { Drosophila } \\
\text { Genomics } \\
\text { Resource } \\
\text { Center }\end{array}$ & $\begin{array}{l}\text { FLYB:FBcl01681 } \\
45\end{array}$ & $\begin{array}{l}\text { FlyBase } \\
\text { symbol: LD25644 }\end{array}$ \\
\hline $\begin{array}{l}\text { gene }(D . \\
\text { melanog } \\
\text { aste })\end{array}$ & slif (cDNA) & $\begin{array}{l}\text { Drosophila } \\
\text { Genomics } \\
\text { Resource } \\
\text { Center }\end{array}$ & $\begin{array}{l}\text { FLYB:FBcl01676 } \\
22\end{array}$ & $\begin{array}{l}\text { FlyBase } \\
\text { symbol: LD37241 }\end{array}$ \\
\hline $\begin{array}{l}\text { gene }(D . \\
\text { melanog } \\
\text { aste) }\end{array}$ & $\begin{array}{l}\text { CG13248 (cD } \\
\text { NA) }\end{array}$ & $\begin{array}{l}\text { Drosophila } \\
\text { Genomics } \\
\text { Resource } \\
\text { Center }\end{array}$ & $\begin{array}{l}\text { FLYB:FBcl07187 } \\
46\end{array}$ & $\begin{array}{l}\text { FlyBase } \\
\text { symbol: FI04531 }\end{array}$ \\
\hline $\begin{array}{l}\text { gene } \\
\text { (Homo-s } \\
\text { apiens) }\end{array}$ & SLC38A3 & $\begin{array}{l}\text { NCBI } \\
\text { database }\end{array}$ & Gene ID: 10991 & $\begin{array}{l}\text { Synthesized from } \\
\text { GENEWIZ, China. }\end{array}$ \\
\hline $\begin{array}{l}\text { genetic } \\
\text { reagent } \\
(D . \\
\text { melanog } \\
\text { aster })\end{array}$ & $\operatorname{tadr}^{\text {RNAi1 }}$ & $\begin{array}{l}\text { Vienna } \\
\text { Drosophila } \\
\text { Resource } \\
\text { Center }\end{array}$ & $\begin{array}{l}\text { BDSC:v330472 } \\
\text { RRID:FlyBase_F } \\
\text { Bst0492187 }\end{array}$ & $\begin{array}{l}\mathrm{P}\{\mathrm{VSH} 330472\} \text { attP4 } \\
0\end{array}$ \\
\hline $\begin{array}{l}\text { cell line } \\
(D . \\
\text { melanog } \\
\text { aste })\end{array}$ & $\mathrm{S} 2$ & This paper & $\begin{array}{l}\text { FLYB:FBtc00001 } \\
81 ; \\
\text { RRID:CVCL_Z99 } \\
2\end{array}$ & $\begin{array}{l}\text { Cell line maintained } \\
\text { in N. Perrimon lab; } \\
\text { FlyBase symbol: } \\
\text { S2-DRSC. }\end{array}$ \\
\hline antibody & $\begin{array}{l}\text { anti-24B10(Mo } \\
\text { use } \\
\text { monoclonal) }\end{array}$ & DHSB & $\begin{array}{l}\text { RRID:AB_52816 } \\
1\end{array}$ & $\mathrm{IF}(1: 100)$ \\
\hline
\end{tabular}




\begin{tabular}{|c|c|c|c|c|}
\hline antibody & $\begin{array}{l}\text { anti-CSP(Mou } \\
\text { se } \\
\text { monoclonal) }\end{array}$ & DHSB & $\begin{array}{l}\text { RRID:AB_52818 } \\
3\end{array}$ & $\operatorname{IF}(1: 100)$ \\
\hline antibody & $\begin{array}{l}\text { anti-RFP(Ratm } \\
\text { onoclonal) }\end{array}$ & Chromotek & $\begin{array}{l}\text { RRID:AB_23360 } \\
64\end{array}$ & $\operatorname{IF}(1: 200)$ \\
\hline antibody & $\begin{array}{l}\text { anti-GFP(Rabb } \\
\text { it polyclonal) }\end{array}$ & Invitrogen & $\begin{array}{l}\text { RRID:AB_22156 } \\
9\end{array}$ & IF(1:200) \\
\hline antibody & $\begin{array}{l}\text { anti-LOVIT(Rat } \\
\text { polyclonal) }\end{array}$ & $\begin{array}{l}\text { (Xu and } \\
\text { Wang, 2019) }\end{array}$ & & $\operatorname{IF}(1: 100)$ \\
\hline antibody & $\begin{array}{l}\text { anti-Hdc(Rabbi } \\
t \text { polyclonal) }\end{array}$ & This paper & & $\mathrm{IF}(1: 50)$ \\
\hline antibody & $\begin{array}{l}\text { anti-Ebony(Ra } \\
\text { bbit } \\
\text { polyclonal) }\end{array}$ & $\begin{array}{l}\text { University of } \\
\text { Wisconsin }\end{array}$ & & $\operatorname{IF}(1: 200)$ \\
\hline antibody & $\begin{array}{l}\text { anti-Histamine( } \\
\text { Rabbit } \\
\text { polyclonal) }\end{array}$ & ImmunoStar & $\begin{array}{l}\text { RRID:AB_57224 } \\
5\end{array}$ & $\operatorname{IF}(1: 100)$ \\
\hline $\begin{array}{l}\text { recombin } \\
\text { ant DNA } \\
\text { reagent }\end{array}$ & $p T r p-G F P$ & This paper & & $\begin{array}{l}\text { Used for generation } \\
\text { of transgenic flies } \\
\text { (maintained in } \mathrm{T} \text {. } \\
\text { Wang lab) }\end{array}$ \\
\hline $\begin{array}{l}\text { recombin } \\
\text { ant DNA } \\
\text { reagent }\end{array}$ & $\begin{array}{l}p T r p-G F P-t a d \\
r\end{array}$ & This paper & & $\begin{array}{l}\text { Used for generation } \\
\text { of transgenic flies } \\
\text { (maintained in } \mathrm{T} \text {. } \\
\text { Wang lab) }\end{array}$ \\
\hline $\begin{array}{l}\text { recombin } \\
\text { ant DNA } \\
\text { reagent }\end{array}$ & $\begin{array}{l}\text { pTrp-tadr-GF } \\
P\end{array}$ & This paper & & $\begin{array}{l}\text { Used for generation } \\
\text { of transgenic flies } \\
\text { (maintained in } \mathrm{T} \text {. } \\
\text { Wang lab) }\end{array}$ \\
\hline
\end{tabular}




\begin{tabular}{|c|c|c|c|c|}
\hline $\begin{array}{l}\text { recombin } \\
\text { ant DNA } \\
\text { reagent }\end{array}$ & pTrp-tadr & This paper & & $\begin{array}{l}\text { Used for generation } \\
\text { of transgenic flies } \\
\text { (maintained in } \mathrm{T} \text {. } \\
\text { Wang lab) }\end{array}$ \\
\hline $\begin{array}{l}\text { sequence } \\
\text {-based } \\
\text { reagent }\end{array}$ & $\begin{array}{l}\text { sgRNA1 (for } \\
\text { tadr }^{2} \text { mutant) }\end{array}$ & This paper & sgRNAs & $\begin{array}{l}\text { GTGCCTGCGCTG } \\
\text { CCCTGGCG }\end{array}$ \\
\hline $\begin{array}{l}\text { sequence } \\
\text {-based } \\
\text { reagent }\end{array}$ & $\begin{array}{l}\text { sgRNA2 (for } \\
\text { tadr }^{2} \text { mutant) }\end{array}$ & This paper & sgRNAs & $\begin{array}{l}\text { TTTTAAGCGCCG } \\
\text { TCGGCTGG }\end{array}$ \\
\hline $\begin{array}{l}\text { sequence } \\
\text {-based } \\
\text { reagent }\end{array}$ & $\begin{array}{l}\text { forward primer } \\
\text { (for tadr } \\
\text { mutant) }\end{array}$ & This paper & PCR primers & $\begin{array}{l}\text { CAATGGCAGGTG } \\
\text { GGAGTTAGG }\end{array}$ \\
\hline $\begin{array}{l}\text { sequence } \\
\text {-based } \\
\text { reagent }\end{array}$ & $\begin{array}{l}\text { reverse primer } \\
\text { (for } t a d r^{2} \\
\text { mutant) }\end{array}$ & This paper & PCR primers & $\begin{array}{l}\text { TTAGAGTCGCCG } \\
\text { TGAATCGTC }\end{array}$ \\
\hline $\begin{array}{l}\text { sequence } \\
\text {-based } \\
\text { reagent }\end{array}$ & $\begin{array}{l}\text { sgRNA (for } \\
\text { GFP-tadr } \\
\text { knock-in) }\end{array}$ & This paper & sgRNAs & $\begin{array}{l}\text { ACAACAACGACA } \\
\text { ATGTCGAG }\end{array}$ \\
\hline $\begin{array}{l}\text { peptide, } \\
\text { recombin } \\
\text { ant } \\
\text { protein }\end{array}$ & $\begin{array}{l}\text { Hdc peptide } \\
(D . \\
\text { melanogaste })\end{array}$ & This paper & $\begin{array}{l}\text { synthesized by } \\
\text { ChinaPeptides } \\
\text { (Soochow, } \\
\text { China) }\end{array}$ & $\begin{array}{l}\text { CDFKEYRQRGKE } \\
\text { MVDY }\end{array}$ \\
\hline $\begin{array}{l}\text { chemical } \\
\text { compoun } \\
\text { d, drug }\end{array}$ & {$\left[{ }^{3} \mathrm{H}\right]$-Histidine } & $\begin{array}{l}\text { American } \\
\text { radiolabeled } \\
\text { chemicals }\end{array}$ & ART 0234 & $30-60 \mathrm{Ci} / \mathrm{mM}$ \\
\hline $\begin{array}{l}\text { chemical } \\
\text { compoun } \\
\text { d, drug }\end{array}$ & $\begin{array}{l}{\left[{ }^{3} \mathrm{H}\right] \text {-Histamin }} \\
\mathrm{e}\end{array}$ & $\begin{array}{l}\text { American } \\
\text { radiolabeled } \\
\text { chemicals }\end{array}$ & ART 1432 & $10-40 \mathrm{Ci} / \mathrm{mM}$ \\
\hline $\begin{array}{l}\text { chemical } \\
\text { compoun } \\
\text { d, drug }\end{array}$ & {$\left[{ }^{3} \mathrm{H}\right]-\beta$-alanine } & $\begin{array}{l}\text { American } \\
\text { radiolabeled } \\
\text { chemicals }\end{array}$ & ART 0205 & 30-60 Ci/mM \\
\hline
\end{tabular}




\begin{tabular}{|l|l|l|l|l|}
\hline $\begin{array}{l}\text { software, } \\
\text { algorithm }\end{array}$ & $\begin{array}{l}\text { GraphPad } \\
\text { Prism software }\end{array}$ & $\begin{array}{l}\text { GraphPad } \\
\text { Prism } \\
\text { (https://graph } \\
\text { pad.com) }\end{array}$ & $\begin{array}{l}\text { RRID:SCR_0158 } \\
07\end{array}$ & Version 7.0.0 \\
\hline $\begin{array}{l}\text { software, } \\
\text { algorithm }\end{array}$ & $\begin{array}{l}\text { ImageJ } \\
\text { software }\end{array}$ & $\begin{array}{l}\text { ImageJ } \\
\text { (http://imagej. } \\
\text { nih.gov/ij/) }\end{array}$ & $\begin{array}{l}\text { RRID:SCR_0030 } \\
70\end{array}$ & \\
\hline
\end{tabular}

Fly Stocks and Cultivation

The $T I\{T \mid\} H d c^{\text {attP }}, H d c^{P 217}$, and M(vas-int.Dm) ZH-2A;M(3xP3-RFP.attP) ZH-86Fb flies were provided by the Bloomington Drosophila Stock Center (https://bdsc.indiana.edu). The tdar RNAit line ( $\mathrm{P}\{\mathrm{VSH} 330472\} a t t P 40)$ was obtained from the Vienna Drosophila Resource Center (https://stockcenter.vdrc.at). The transgenic RNAi lines for the in vivo transporter screen were obtained from the TsingHua Fly Center (http://fly.redbux.cn), the Bloomington Drosophila Stock Center, and the Vienna Drosophila Resource Center. The $w^{1118}$, nos-Cas9, GMR-gal4, and Repo-Gal4 flies were maintained in the lab of Dr. T. Wang at the National Institute of Biological Sciences, Beijing, China. Flies were maintained in 12-h-light-12-h-dark cycles with 2000 lux illumination at $25^{\circ} \mathrm{C}$, except when mentioned differently in the text.

\section{Generation of tadr mutant and knock-in flies}

The $t a d r^{2}$ mutation was generated using the Cas9/sgRNA system (Xu et al., 2015). Briefly, two pairs of guide RNAs targeting the tadr locus were designed (sgRNA1: GTGCCTGCGCTGCCCTGGCG, sgRNA2: TTTTAAGCGCCGTCGGCTGG) and cloned into the U6b-sgRNA-short vector. The plasmids were injected into the embryos of nos-Cas 9 flies, and deletions were identified by PCR using the following primers: forward primer 5'CAATGGCAGGTGGGAGTTAGG-3' and reverse primer 5'TTAGAGTCGCCGTGAATCGTC-3'. The GFP-tadr knock-in flies were generated as shown in Figure S3. Briefly, a sgDNA sequence (ACAACAACGACAATGTCGAG) was designed and cloned into the U6b-sgRNA-short vector. tadr genomic DNA (from 747 base pairs (bp) upstream of the transcription starting site to $893 \mathrm{bp}$ downstream of the transcription termination site) was subcloned into a donor vector. GFP-tag sequence was then inserted at the end of the upstream fragment sequence. The two plasmids were co-injected into the embryos of nos-Cas9 flies. The GFP-tadr flies were finally confirmed by PCR of genomic DNA using the following primers: forward primer 5'- ATGGTGAGCAAGGGCGAGG -3' and reverse primer 5'- GAATACCCACACACATGCCAATCA -3'. Both tadr ${ }^{2}$ and GFP-tadr flies were backcrossed to wild-type flies $\left(w^{1118}\right)$ for two generations before preforming experiments.

\section{Generation of plasmid constructs and transgenic flies}

Amino acid transporters, including tadr, slif, and CG13248 cDNA sequences were amplified from LD25644, LD37241, and FI04531 cDNA clones obtained from DGRC (Drosophila Genomics Resource Center, Bloomington, IN, USA). The human SLC38A3 cDNA sequences 
were synthesized from GENEWIZ, China. Their entire CDS sequences were subcloned into the pCDNA3 vector (Invitrogen, Carlsbad, USA) for expression in HEK293T cells or PIB vector (Invitrogen, Carlsbad, USA) for expression in S2 cells. To expressing cDNAs in the photoreceptor cells, a 1.7-kb genomic DNA fragment $\left(-1656\right.$ to +176 base pairs $5^{\prime}$ to the transcription start site) of trp locus substituted the UAS sequence of $p U A S T$-attB vector to generate the $p$ Trp-attB vector (Bischof et al., 2007; Li and Montell, 2000). To construct pTrp-tadr, pTrp-GFP-tadr and pTrp-tadr-GFP, the entire coding region of tadr was subcloned into the $p$ Trp-attB vector with N-GFP or C-GFP-tags. To construct $p$ Trp-Hdc-mCherry, the entire CDS sequence of $H d c$ with a C-terminal mcherry tag was cloned into the $p$ Trp-attB vector. To construct UAS-tadr, UAS-CG13248, UAS-Slif and UAS-SLC38A3 plasmids, cDNAs of tadr, CG13248, Slif and SLC38A3 were amplified, and subcloned to UAST-attB vector. We produced a $\operatorname{tadr}^{R N A 2 i}$ line as described (Ni et al., 2011) by designing a 21-nucleotide short hairpin RNA sequences (GCCACAAGATGAGCAGCAAAT), and cloning them into a VALIUM20 vector. These constructs were injected into $M$ (vas-int.Dm) ZH-2A;M(3xP3-RFP.attP) $Z H-86 F b$ embryos, and transformants were identified on the basis of eye color. The 3xP3-RFP and $w+$ markers were removed by crossing with $P(C r e)$ flies.

\section{Generation of anti-Hdc antibody}

A Hdc peptide CDFKEYRQRGKEMVDY was synthesized by ChinaPeptides (Soochow, China), linked with BSA, and injected into rats by the Antibody Center at NIBS to generate anti-Hdc antibodies. The animal work for generating the antisera was conducted following the National Guidelines for Housing and Care of Laboratory Animals in China, and performed in accordance with institutional regulations after approval by the IACUC at NIBS (Reference\# NIBS2016R0001).

\section{Electroretinogram recordings}

ERGs were recorded as described (Xu et al., 2015). Briefly, two glass microelectrodes were filled with Ringer's solution, and placed on the surfaces of the compound eye and thorax (one each surface). The light intensity was $\sim 0.3 \mathrm{~mW} / \mathrm{cm}^{2}$, and the wavelength was $\sim 550 \mathrm{~nm}$ (source light was filtered using a FSR-OG550 filter). The electoral signals were amplified with a Warner electrometer IE-210, and were recorded with a MacLab/4 s A/D converter and Clampex 10.2 program (Warner Instruments, Hamden, USA). All recordings were carried out at $25^{\circ} \mathrm{C}$.

\section{Histidine, $\beta$-alanine, histamine, and carcinine uptake assay}

$\left[{ }^{3} \mathrm{H}\right]$-Histidine (30-60 Ci/mM, American radiolabeled chemicals, Saint Louis, USA), $\beta$-alanine, $\left[3^{3} \mathrm{H}(\mathrm{N})\right](30-60 \mathrm{Ci} / \mathrm{mM}$, American radiolabeled chemicals, Saint Louis, USA), and Histamine, histamine [ring, Methylenes- ${ }^{3} \mathrm{H}(\mathrm{N})$ ] dihydrochloride, (10-40 Ci/mM, American radiolabeled chemicals, Saint Louis, USA) uptake were measured as described (Han et al., 2017). Briefly, S2 cells were cultured in Schneider's Drosophila medium with 10\% Fetal Bovine Serum (Gibco, Carlsbad,USA) in 12-well plates, and transfected with vigofect reagent (Vigorous Biotechnology, Beijing, China). The transfected cells were washed with $1 \mathrm{~mL}$ extracellular fluid (ECF) buffer consisting of $120 \mathrm{mM} \mathrm{NaCl}, 25 \mathrm{mM} \mathrm{NaHCO}_{3}, 3 \mathrm{mM} \mathrm{KCl}, 1.4 \mathrm{mM} \mathrm{CaCl}_{2}, 1.2 \mathrm{mM}$ $\mathrm{MgSO}_{4}, 0.4 \mathrm{mM} \mathrm{K}_{2} \mathrm{HPO}_{4}, 10 \mathrm{mM}$ D-glucose, and $10 \mathrm{mM}$ Hepes (pH 7.4) at $37^{\circ} \mathrm{C}$. Uptake 
assays were initiated by applying $200 \mu \mathrm{L}$ DMEM (for histidine uptake) or ECF buffer (for histamine and $\beta$-alanine uptake) at $37^{\circ} \mathrm{C}$. After 10 or 30 minutes, the reaction was terminated by removing the solution, and cells were washed with $1 \mathrm{~mL}$ ice-cold ECF buffer. The cells were then solubilized in $1 \mathrm{~N} \mathrm{NaOH}$ and subsequently neutralized. An aliquot was taken to measure radioactivity and protein content using a liquid scintillation counter and a DC protein assay kit (Bio-rad, USA), respectively. To perform histidine competition assays, $\left[{ }^{3} \mathrm{H}\right]$-histidine $(30-60$ $\mathrm{Ci} / \mathrm{mM}, 2.5 \mu \mathrm{M})$ in combination with L-amino acid including serine, alanine, cysteine, glutamine, asparagine, arginine and lysine, at higher concentration $(0.5 \mathrm{mM})$ were added into DMEM buffer. Carcinine was added to the medium to yield a final concentration of $20 \mu \mathrm{M}$. After incubation for $3 \mathrm{~h}$, S2 cells were transferred to poly-L-lysinecoated slices, fixed with $4 \%$ paraformaldehyde, and incubated with rabbit anti-carcinine/histamine antibodies (1:100, ImmunoStar, USA). Goat anti-rabbit IgG conjugated to Alexa 488 (1:500, Invitrogen, CA) was used as secondary antibodies, and images were recorded with a Nikon A1-R confocal microscope.

\section{Immunohistochemistry}

Fly head sections (10- $\mu \mathrm{m}$ thick) were prepared from adults that were frozen in OCT medium (Tissue-Tek, Torrance, USA). Immunolabeling was performed on cryosections sections with mouse anti-24B10 (1:100, DSHB), rat anti-LOVIT (1:100) (Xu and Wang, 2019), or anti-CSP (1:100, DSHB), rat anti-RFP (1:200, Chromotek, Germany), rabbit anti-Hdc (1:50), rabbit anti-GFP (1:200, Invitrogen, USA), and rabbit anti-Ebony (1:200, lab of Dr. S. Carroll, University of Wisconsin, Madison, USA) as primary antibodies. For histamine immunolabeling, the rabbit anti-histamine (1:100, ImmunoStar, USA) antibody was pre-adsorbed with carcinine, as previously reported (Xu et al., 2015). Goat anti-rabbit lgG conjugated to Alexa 488 (1:500, Invitrogen, USA), goat anti-mouse IgG conjugated to Alexa 488 (1:500, Invitrogen, USA), goat anti- rabbit IgG conjugated to Alexa 568 (1:500, Invitrogen, USA), and goat anti-rat IgG conjugated to Alexa 647 (1:500, Invitrogen, USA) were used as secondary antibodies. The images were recorded with a Zeiss 800 confocal microscope.

\section{The phototaxis assay}

Flies were dark adapted for 15 min before phototaxis assay, as described (Xu et al., 2015). A white light source (with an intensity of $\sim 6,000$ lux) was used, and phototaxis index was calculated by dividing the total number of flies by the number of flies that walked above the mark. Five groups of flies were collected for each genotype, and three repeats were made for each group. Each group contained at least 20 flies. Results were expressed as the mean of the mean values for the four groups.

\section{Transmission Electron Microscopy}

To visualize Drosophila retina ultrastructure, adult fly heads were dissected, fixed, dehydrated, and embedded in LR White resin (Electron Microscopy Sciences) as described (Xu et al., 2015). Thin sections (80 $\mathrm{nm}$ ) at a depth of 30-40 $\mu \mathrm{m}$ were prepared, and examined using a JEM-1400 transmission electron microscope (JEOL, Tokyo, Japan) equipped with a Gatan CCD $(4 \mathrm{k} \times 3.7 \mathrm{k}$ pixels, USA). TEM of photoreceptor terminals was performed as described 
(Xu and Wang, 2019). Adult fly heads were dissected and fixed in 4\% PFA. The laminas were further dissected by removing retinas, followed by fine fixation in $1 \%$ osmium tetroxide for 1.5 $\mathrm{h}$ at $4^{\circ} \mathrm{C}$. Thin sections $(80 \mathrm{~nm})$ were stained with uranyl acetate and lead-citrate (Ted Pella) and examined using a JEM-1400 transmission electron microscope (JEOL, Tokyo, Japan) equipped with a Gatan CCD $(4 \mathrm{k} \times 3.7 \mathrm{k}$ pixels, USA).

\section{Liquid chromatography-mass spectrometry (LC-MS)}

LC-MS was performed as previous reported (Han et al., 2017). The Dionex Ultimate 3000 UPLC system was coupled to a TSQ Quantiva Ultra triple-quadrupole mass spectrometer (Thermo Fisher, CA), equipped with a heated electrospray ionization (HESI) probe in negative ion mode. Extracts were separated by a Fusion-RP C18 column $(2 \times 100 \mathrm{~mm}, 2.5 \mu \mathrm{m}$, phenomenex). Data acquired in selected reaction monitoring (SRM) for histamine, carcinine, and $\beta$-alanine with transitions of 112/95.2, 183/95, and 90/72, respectively. Both precursor and fragment ions were collected with resolution of $0.7 \mathrm{FWHM}$. The source parameters are as follows: spray voltage: $3000 \mathrm{~V}$; ion transfer tube temperature: $350{ }^{\circ} \mathrm{C}$; vaporizer temperature: $300{ }^{\circ} \mathrm{C}$; sheath gas flow rate: $40 \mathrm{Arb}$; auxiliary gas flow rate: $20 \mathrm{Arb}$; CID gas: 2.0 mTorr. Data analysis and quantification were performed using the software Xcalibur 3.0.63 (Thermo Fisher, $\mathrm{CA}$ ). Each sample contained 50 Drosophila heads, and the mean values from five samples were calculated.

\section{Quantification and statistical analysis}

All experiments were repeated as indicated in each figure legend. All statistical analyses were performed using GraphPad Prism 7. The variations of data were evaluated as mean \pm SD. The statistical significance of the differences between two groups was measured by the unpaired two-tailed Student's t test, and one-way ANOVA or two-way ANOVA with Tukey's method, two-sided were performed for multi-group comparisons. A value of $p<0.05$ was considered statistically significant (ns, not significant; ${ }^{*} p<0.05$; ${ }^{* *} p<0.01$; ${ }^{* *} p<0.001$ ). P value, standard error of the mean (SD), and number are as indicated in each figure and legend.

\section{Acknowledgments}

We thank the Bloomington Stock Center, Drosophila Genomic Resource Center, the Developmental Studies Hybridoma Bank, Vienna Drosophila Resource Center, TsingHua Fly Center and Dr. S. Carroll for stocks and reagents. We thank Y. Wang and X. Liu for assistance with fly injections. We are tremendously thankful for support provided by the Metabolomics Facility and Image Facility at NIBS. We thank State Key Laboratory of Membrane Biology, Institute of Zoology, Chinese Academy of Science for our Electron Microscopy and we would be grateful to Pengyan Xia for his help of taking EM images. We thank Dr. D. O'Keefe for comments on the manuscript. This work was supported by grants from the National Natural Science Foundation of China (81870693 and 81670891) awarded to T. Wang.

\section{Competing interests}

The authors declare no competing financial interests. 


\begin{tabular}{|c|c|c|}
\hline CG Number & ON/OFF & Description \\
\hline CG4991 & Yes & Amino acid transmembrane transporter activity/SLC36A1 or A2 \\
\hline CG7888 & Yes & Amino acid transmembrane transporter activity/SLC36A1 or A2 \\
\hline CG1139 & Yes & Amino acid transmembrane transporter activity/SLC36A1 or A2 \\
\hline CG8785 & Yes & Amino acid transmembrane transporter activity/SLC36A1 or A2 \\
\hline CG32079 & Yes & Amino acid transmembrane transporter activity/SLC36A1 or A2 \\
\hline CG32081 & Yes & Amino acid transmembrane transporter activity/SLC36A1 or A2 \\
\hline CG16700 & Yes & Amino acid transmembrane transporter activity/SLC36A1 or A4 \\
\hline CG13384 & Yes & Amino acid transmembrane transporter activity/SLC $36 \mathrm{~A} 1$ or $\mathrm{A} 4$ \\
\hline CG43693 & Yes & Amino acid transmembrane transporter activity/SLC36A1 or A4 \\
\hline polyph & Yes & Amino acid transmembrane transporter activity/SLC36A1 or A2 \\
\hline path & Yes & Amino acid transmembrane transporter activity/SLC36A1 or A2 \\
\hline mah & Yes & Amino acid transmembrane transporter activity/SLC36A1 or A2 \\
\hline CG30394 & Yes & Amino acid transmembrane transporter activity/SLC38A10 \\
\hline CG13743 & Yes & Amino acid transmembrane transporter activity/SLC38A11 \\
\hline CG13248 & Yes & Amino acid transmembrane transporter activity/SLC7A4 \\
\hline slif & Yes & Amino acid transmembrane transporter activity/SLC7A1 or A2 \\
\hline CG12773 & Yes & Amino acid transmembrane transporter activity/SLC12A8 \\
\hline NKCC & Yes & Amino acid transmembrane transporter activity/SLC12A3 \\
\hline ChT & Yes & Amino acid transmembrane transporter activity/SLC5A7 \\
\hline NAAT1 & Yes & Amino acid transmembrane transporter activity/SLC6A7 or A9 \\
\hline CG15279 & Yes & L-amino acid transmembrane transporter activity/SLC6A7 or A9 \\
\hline CG4476 & Yes & L-amino acid transmembrane transporter activity/SLC6A7 or A9 \\
\hline CG1698 & Yes & L-amino acid transmembrane transporter activity/SLC6A7 or A9 \\
\hline List & Yes & L-amino acid transmembrane transporter activity/SLC6A7 \\
\hline$J h I-21$ & Yes & L-amino acid transmembrane transporter activity/SLC6A5 \\
\hline mnd & Yes & L-amino acid transmembrane transporter activity/SLC7A6 or A7 \\
\hline$g b$ & Yes & L-amino acid transmembrane transporter activity/SLC7A6 or A7 \\
\hline CG1607 & Yes & L-amino acid transmembrane transporter activity/SLC7A8 \\
\hline$s b m$ & Yes & L-amino acid transmembrane transporter activity/SLC7A9 \\
\hline Eaat1 & Yes & L-amino acid transmembrane transporter activity/SLC7A3 \\
\hline Eaat2 & Yes & L-amino acid transmembrane transporter activity/SLC7A2 \\
\hline GC1 & Yes & L-glutamate transmembrane transport/SLC25A18 \\
\hline $\operatorname{tad} r$ & No & Cationic amino acid transporter/SLC7A4 or SLC7A1 \\
\hline VGlut & Yes & Vesicular glutamate transporter/SLC17A7 \\
\hline Gat & Yes & GABA transporter activity/SLC6A1 \\
\hline$k c c$ & Yes & Potassium:chloride symporter activity/SLC12A4 \\
\hline Sfxn1-3 & Yes & Serine transmembrane transporter activity/SFXN1 \\
\hline VGAT & Yes & Vesicular GABA transporter activity/SLC32A1 \\
\hline Ncc69 & Yes & Sodium:potassium:chloride symporter activity/SLC12A1 or A2 \\
\hline CG1265 & Yes & Lysosomal amino acid transporter/SLC66A3 \\
\hline CG3792 & Yes & Lysosomal amino acid transporter/SLC66 \\
\hline CG13784 & Yes & Lysosomal amino acid transporter/SLC66A2 \\
\hline
\end{tabular}




\section{References}

Andersen, J., Kristensen, A.S., Bang-Andersen, B., and Strømgaard, K. (2009). Recent advances in the understanding of the interaction of antidepressant drugs with serotonin and norepinephrine transporters. Chemical communications (Cambridge, England), 3677-3692.

Aust, S., Brüsselbach, F., Pütz, S., and Hovemann, B.T. (2010). Alternative tasks of Drosophila tan in neurotransmitter recycling versus cuticle sclerotization disclosed by kinetic properties. The Journal of biological chemistry 285, 20740-20747.

Baldan, L.C., Williams, K.A., Gallezot, J.D., Pogorelov, V., Rapanelli, M., Crowley, M., Anderson, G.M., Loring, E., Gorczyca, R., Billingslea, E., et al. (2014). Histidine decarboxylase deficiency causes tourette syndrome: parallel findings in humans and mice. Neuron 81, 77-90.

Behnia, R., and Desplan, C. (2015). Visual circuits in flies: beginning to see the whole picture. Current opinion in neurobiology 34, 125-132.

Bekkers, J.M. (1993). Enhancement by histamine of NMDA-mediated synaptic transmission in the hippocampus. Science (New York, NY) 261, 104-106.

Bellipanni, G., Rink, E., and Bally-Cuif, L. (2002). Cloning of two tryptophan hydroxylase genes expressed in the diencephalon of the developing zebrafish brain. Mechanisms of development 119 Suppl 1, S215-220.

Bischof, J., Maeda, R.K., Hediger, M., Karch, F., and Basler, K. (2007). An optimized transgenesis system for Drosophila using germ-line-specific phiC31 integrases. Proceedings of the National Academy of Sciences of the United States of America 104, 3312-3317.

Borycz, J., Borycz, J.A., Loubani, M., and Meinertzhagen, I.A. (2002). tan and ebony genes regulate a novel pathway for transmitter metabolism at fly photoreceptor terminals. The Journal of neuroscience : the official journal of the Society for Neuroscience 22, 10549-10557.

Borycz, J., Vohra, M., Tokarczyk, G., and Meinertzhagen, I.A. (2000). The determination of histamine in the Drosophila head. Journal of neuroscience methods 101, 141-148.

Bröer, S. (2014). The SLC38 family of sodium-amino acid co-transporters. Pflugers Archiv : European journal of physiology 466, 155-172.

Bröer, S., and Bröer, A. (2017). Amino acid homeostasis and signalling in mammalian cells and organisms. The Biochemical journal 474, 1935-1963.

Broix, L., Turchetto, S., and Nguyen, L. (2021). Coordination between Transport and Local Translation in Neurons. Trends in cell biology 31, 372-386.

Burg, M.G., Sarthy, P.V., Koliantz, G., and Pak, W.L. (1993). Genetic and molecular identification of a Drosophila histidine decarboxylase gene required in photoreceptor transmitter synthesis. The EMBO journal 12, 911-919.

Busch, A.E., Karbach, U., Miska, D., Gorboulev, V., Akhoundova, A., Volk, C., Arndt, P., Ulzheimer, J.C., Sonders, M.S., Baumann, C., et al. (1998). Human neurons express the polyspecific cation transporter hOCT2, which translocates monoamine neurotransmitters, amantadine, and memantine. Molecular pharmacology 54, 342-352.

Cartier, E.A., Parra, L.A., Baust, T.B., Quiroz, M., Salazar, G., Faundez, V., Egaña, L., and Torres, G.E. (2010). A biochemical and functional protein complex involving dopamine synthesis and transport into synaptic vesicles. The Journal of biological chemistry 285, 1957-1966.

Chaturvedi, R., Luan, Z., Guo, P., and Li, H.S. (2016). Drosophila Vision Depends on Carcinine Uptake by an Organic Cation Transporter. Cell reports 14, 2076-2083.

Chaturvedi, R., Reddig, K., and Li, H.S. (2014). Long-distance mechanism of neurotransmitter recycling 
mediated by glial network facilitates visual function in Drosophila. Proceedings of the National Academy of Sciences of the United States of America 111, 2812-2817. Chen, R., Zou, Y., Mao, D., Sun, D., Gao, G., Shi, J., Liu, X., Zhu, C., Yang, M., Ye, W., et al. (2014). The general amino acid control pathway regulates mTOR and autophagy during serum/glutamine starvation. The Journal of cell biology 206, 173-182.

Colombani, J., Raisin, S., Pantalacci, S., Radimerski, T., Montagne, J., and Léopold, P. (2003). A nutrient sensor mechanism controls Drosophila growth. Cell 114, 739-749.

Deshpande, S.A., Freyberg, Z., Lawal, H.O., and Krantz, D.E. (2020). Vesicular neurotransmitter transporters in Drosophila melanogaster. Biochimica et biophysica acta Biomembranes 1862, 183308. Ercan-Sencicek, A.G., Stillman, A.A., Ghosh, A.K., Bilguvar, K., O'Roak, B.J., Mason, C.E., Abbott, T., Gupta, A., King, R.A., Pauls, D.L., et al. (2010). L-histidine decarboxylase and Tourette's syndrome. The New England journal of medicine 362, 1901-1908.

Gengs, C., Leung, H.T., Skingsley, D.R., lovchev, M.I., Yin, Z., Semenov, E.P., Burg, M.G., Hardie, R.C., and Pak, W.L. (2002). The target of Drosophila photoreceptor synaptic transmission is a histamine-gated chloride channel encoded by ort (hclA). The Journal of biological chemistry 277, 42113-42120.

Gisselmann, G., Pusch, H., Hovemann, B.T., and Hatt, H. (2002). Two cDNAs coding for histamine-gated ion channels in D. melanogaster. Nature neuroscience 5, 11-12.

Haas, H., and Panula, P. (2003). The role of histamine and the tuberomamillary nucleus in the nervous system. Nature reviews Neuroscience 4, 121-130.

Haas, H.L., Sergeeva, O.A., and Selbach, O. (2008). Histamine in the nervous system. Physiological reviews $88,1183-1241$.

Han, Y., Xiong, L., Xu, Y., Tian, T., and Wang, T. (2017). The $\beta$-alanine transporter BalaT is required for visual neurotransmission in Drosophila. elife 6.

Hansson, S.R., Mezey, E., and Hoffman, B.J. (1999). Serotonin transporter messenger RNA expression in neural crest-derived structures and sensory pathways of the developing rat embryo. Neuroscience 89, 243-265.

Hardie, R.C. (1989). A histamine-activated chloride channel involved in neurotransmission at a photoreceptor synapse. Nature 339, 704-706.

Huang, Z.L., Qu, W.M., Li, W.D., Mochizuki, T., Eguchi, N., Watanabe, T., Urade, Y., and Hayaishi, O. (2001). Arousal effect of orexin A depends on activation of the histaminergic system. Proceedings of the National Academy of Sciences of the United States of America 98, 9965-9970.

Kanai, Y. (2021). Amino acid transporter LAT1 (SLC7A5) as a molecular target for cancer diagnosis and therapeutics. Pharmacology \& therapeutics, 107964.

Karl, P.I., Tkaczevski, H., and Fisher, S.E. (1989). Characteristics of histidine uptake by human placental microvillous membrane vesicles. Pediatric research 25, 19-26.

Klaips, C.L., Jayaraj, G.G., and Hartl, F.U. (2018). Pathways of cellular proteostasis in aging and disease. The Journal of cell biology 217, 51-63.

Kurian, M.A., Gissen, P., Smith, M., Heales, S., Jr., and Clayton, P.T. (2011). The monoamine neurotransmitter disorders: an expanding range of neurological syndromes. The Lancet Neurology 10, 721-733.

Lebrand, C., Cases, O., Adelbrecht, C., Doye, A., Alvarez, C., El Mestikawy, S., Seif, I., and Gaspar, P. (1996). Transient uptake and storage of serotonin in developing thalamic neurons. Neuron 17 , 823-835.

Li, H.S., and Montell, C. (2000). TRP and the PDZ protein, INAD, form the core complex required for 
retention of the signalplex in Drosophila photoreceptor cells. The Journal of cell biology 150, 1411-1422.

Lim, J., and Yue, Z. (2015). Neuronal aggregates: formation, clearance, and spreading. Developmental cell 32, 491-501.

Martin, C.A., and Krantz, D.E. (2014). Drosophila melanogaster as a genetic model system to study neurotransmitter transporters. Neurochemistry international 73, 71-88.

McKinney, J., Teigen, K., Frøystein, N.A., Salaün, C., Knappskog, P.M., Haavik, J., and Martínez, A. (2001). Conformation of the substrate and pterin cofactor bound to human tryptophan hydroxylase. Important role of Phe313 in substrate specificity. Biochemistry 40, 15591-15601.

Melzig, J., Buchner, S., Wiebel, F., Wolf, R., Burg, M., Pak, W.L., and Buchner, E. (1996). Genetic depletion of histamine from the nervous system of Drosophila eliminates specific visual and mechanosensory behavior. Journal of comparative physiology A, Sensory, neural, and behavioral physiology 179, 763-773.

Melzig, J., Burg, M., Gruhn, M., Pak, W.L., and Buchner, E. (1998). Selective histamine uptake rescues photo- and mechanoreceptor function of histidine decarboxylase-deficient Drosophila mutant. The Journal of neuroscience : the official journal of the Society for Neuroscience 18, 7160-7166.

Montell, C., and Rubin, G.M. (1989). Molecular characterization of the Drosophila trp locus: a putative integral membrane protein required for phototransduction. Neuron 2, 1313-1323.

Nałęcz, K.A. (2020). Amino Acid Transporter SLC6A14 (ATB(0,+)) - A Target in Combined Anti-cancer Therapy. Frontiers in cell and developmental biology 8, 594464.

Ni, J.-Q., Zhou, R., Czech, B., Liu, L.-P., Holderbaum, L., Yang-Zhou, D., Shim, H.-S., Tao, R., Handler, D., Karpowicz, P., et al. (2011). A genome-scale shRNA resource for transgenic RNAi in Drosophila. Nat Methods 8, 405-407.

Ni, L., Guo, P., Reddig, K., Mitra, M., and Li, H.S. (2008). Mutation of a TADR protein leads to rhodopsin and Gq-dependent retinal degeneration in Drosophila. The Journal of neuroscience : the official journal of the Society for Neuroscience 28, 13478-13487.

Nicklin, P., Bergman, P., Zhang, B., Triantafellow, E., Wang, H., Nyfeler, B., Yang, H., Hild, M., Kung, C., Wilson, C., et al. (2009). Bidirectional transport of amino acids regulates mTOR and autophagy. Cell 136, 521-534.

Olivero, P., Lozano, C., Sotomayor-Zárate, R., Meza-Concha, N., Arancibia, M., Córdova, C., González-Arriagada, W., Ramírez-Barrantes, R., and Marchant, I. (2018). Proteostasis and Mitochondrial Role on Psychiatric and Neurodegenerative Disorders: Current Perspectives. Neural plasticity 2018, 6798712.

Panula, P., and Nuutinen, S. (2013). The histaminergic network in the brain: basic organization and role in disease. Nature reviews Neuroscience 14, 472-487.

Parmentier, R., Ohtsu, H., Djebbara-Hannas, Z., Valatx, J.L., Watanabe, T., and Lin, J.S. (2002). Anatomical, physiological, and pharmacological characteristics of histidine decarboxylase knock-out mice: evidence for the role of brain histamine in behavioral and sleep-wake control. The Journal of neuroscience : the official journal of the Society for Neuroscience 22, 7695-7711.

Rebsamen, M., Pochini, L., Stasyk, T., de Araújo, M.E., Galluccio, M., Kandasamy, R.K., Snijder, B., Fauster, A., Rudashevskaya, E.L., Bruckner, M., et al. (2015). SLC38A9 is a component of the lysosomal amino acid sensing machinery that controls mTORC1. Nature 519, 477-481.

Ren, Q., Chen, K., and Paulsen, I.T. (2007). TransportDB: a comprehensive database resource for cytoplasmic membrane transport systems and outer membrane channels. Nucleic acids research 35 , 
D274-279.

Richardt, A., Kemme, T., Wagner, S., Schwarzer, D., Marahiel, M.A., and Hovemann, B.T. (2003). Ebony, a novel nonribosomal peptide synthetase for beta-alanine conjugation with biogenic amines in Drosophila. The Journal of biological chemistry 278, 41160-41166.

Richardt, A., Rybak, J., Störtkuhl, K.F., Meinertzhagen, I.A., and Hovemann, B.T. (2002). Ebony protein in the Drosophila nervous system: optic neuropile expression in glial cells. The Journal of comparative neurology 452, 93-102.

Roy, S. (2020). Finding order in slow axonal transport. Current opinion in neurobiology 63, 87-94. Stenesen, D., Moehlman, A.T., and Krämer, H. (2015). The carcinine transporter CarT is required in Drosophila photoreceptor neurons to sustain histamine recycling. eLife 4, e10972.

Stuart, A.E. (1999). From fruit flies to barnacles, histamine is the neurotransmitter of arthropod photoreceptors. Neuron 22, 431-433.

Taguchi, Y., Watanabe, T., Kubota, H., Hayashi, H., and Wada, H. (1984). Purification of histidine decarboxylase from the liver of fetal rats and its immunochemical and immunohistochemical characterization. The Journal of biological chemistry 259, 5214-5221.

Vallee, R.B., and Bloom, G.S. (1991). Mechanisms of fast and slow axonal transport. Annual review of neuroscience $14,59-92$.

Verrey, F., Closs, E.I., Wagner, C.A., Palacin, M., Endou, H., and Kanai, Y. (2004). CATs and HATs: the SLC7 family of amino acid transporters. Pflugers Archiv : European journal of physiology 447, 532-542. Vorobjev, V.S., Sharonova, I.N., Walsh, I.B., and Haas, H.L. (1993). Histamine potentiates $\mathrm{N}$-methyl-D-aspartate responses in acutely isolated hippocampal neurons. Neuron 11, 837-844. Wagner, S., Heseding, C., Szlachta, K., True, J.R., Prinz, H., and Hovemann, B.T. (2007). Drosophila photoreceptors express cysteine peptidase tan. The Journal of comparative neurology 500, 601-611. Walther, D.J., Peter, J.U., Bashammakh, S., Hörtnagl, H., Voits, M., Fink, H., and Bader, M. (2003). Synthesis of serotonin by a second tryptophan hydroxylase isoform. Science (New York, NY) $299,76$. Wang, T., and Montell, C. (2007). Phototransduction and retinal degeneration in Drosophila. Pflugers Archiv : European journal of physiology 454, 821-847.

Wang, Y.C., Lauwers, E., and Verstreken, P. (2017). Presynaptic protein homeostasis and neuronal function. Current opinion in genetics \& development 44, 38-46. Watanabe, T., Taguchi, Y., Shiosaka, S., Tanaka, J., Kubota, H., Terano, Y., Tohyama, M., and Wada, H. (1984). Distribution of the histaminergic neuron system in the central nervous system of rats; a fluorescent immunohistochemical analysis with histidine decarboxylase as a marker. Brain research 295, 13-25.

Wyant, G.A., Abu-Remaileh, M., Wolfson, R.L., Chen, W.W., Freinkman, E., Danai, L.V., Vander Heiden, M.G., and Sabatini, D.M. (2017). mTORC1 Activator SLC38A9 Is Required to Efflux Essential Amino Acids from Lysosomes and Use Protein as a Nutrient. Cell 171, 642-654.e612. Xu, Y., An, F., Borycz, J.A., Borycz, J., Meinertzhagen, I.A., and Wang, T. (2015). Histamine Recycling Is Mediated by CarT, a Carcinine Transporter in Drosophila Photoreceptors. PLoS genetics 11, e1005764. $\mathrm{Xu}, \mathrm{Y}$., and Wang, T. (2016). CULD is required for rhodopsin and TRPL channel endocytic trafficking and survival of photoreceptor cells. Journal of cell science 129, 394-405.

Xu, Y., and Wang, T. (2019). LOVIT Is a Putative Vesicular Histamine Transporter Required in Drosophila for Vision. Cell reports 27, 1327-1333.e1323. 
Figure 1

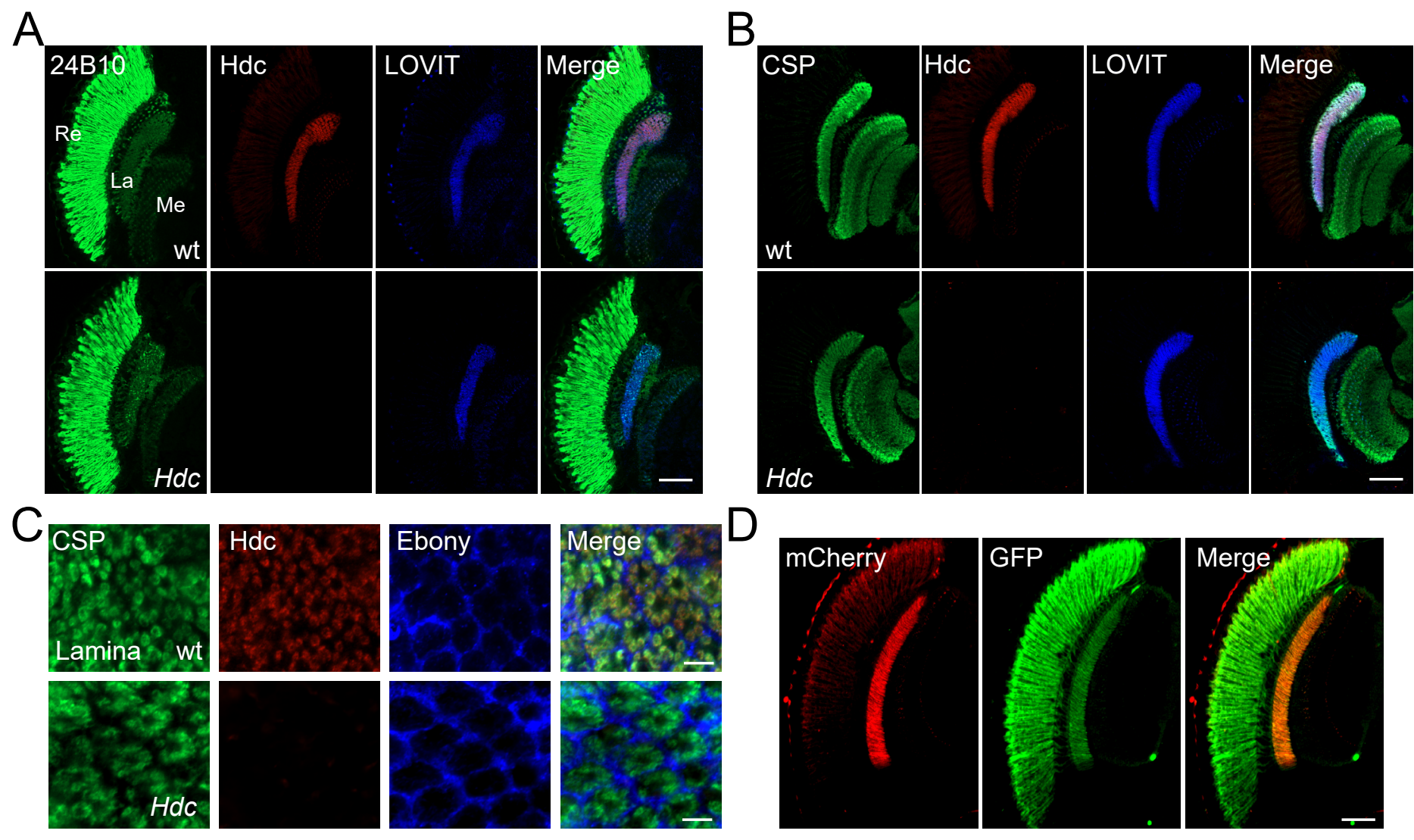


Figure 1-figure supplement 1
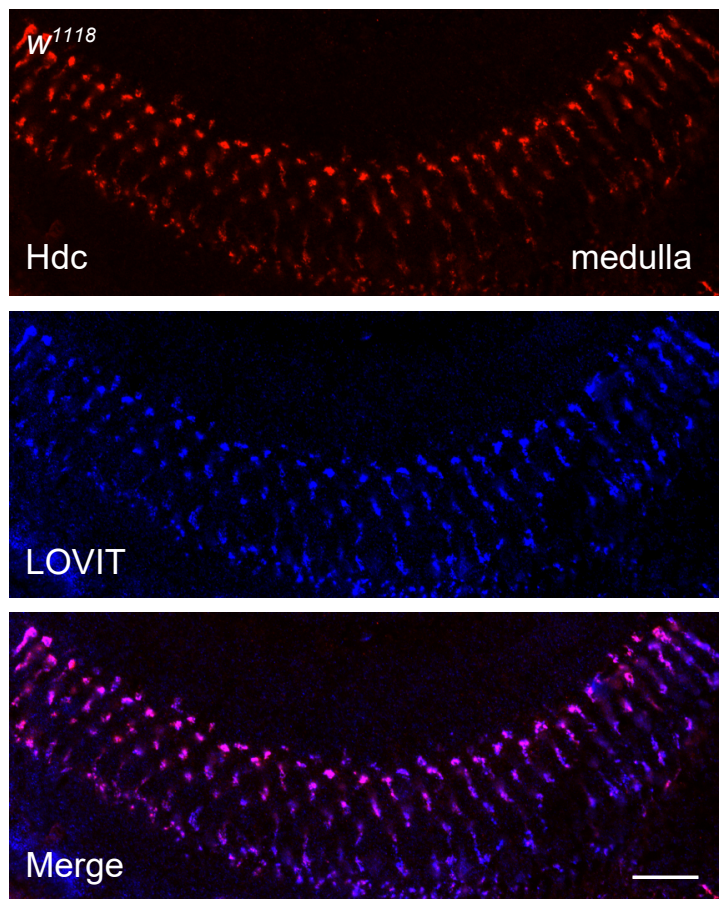
Figure 2
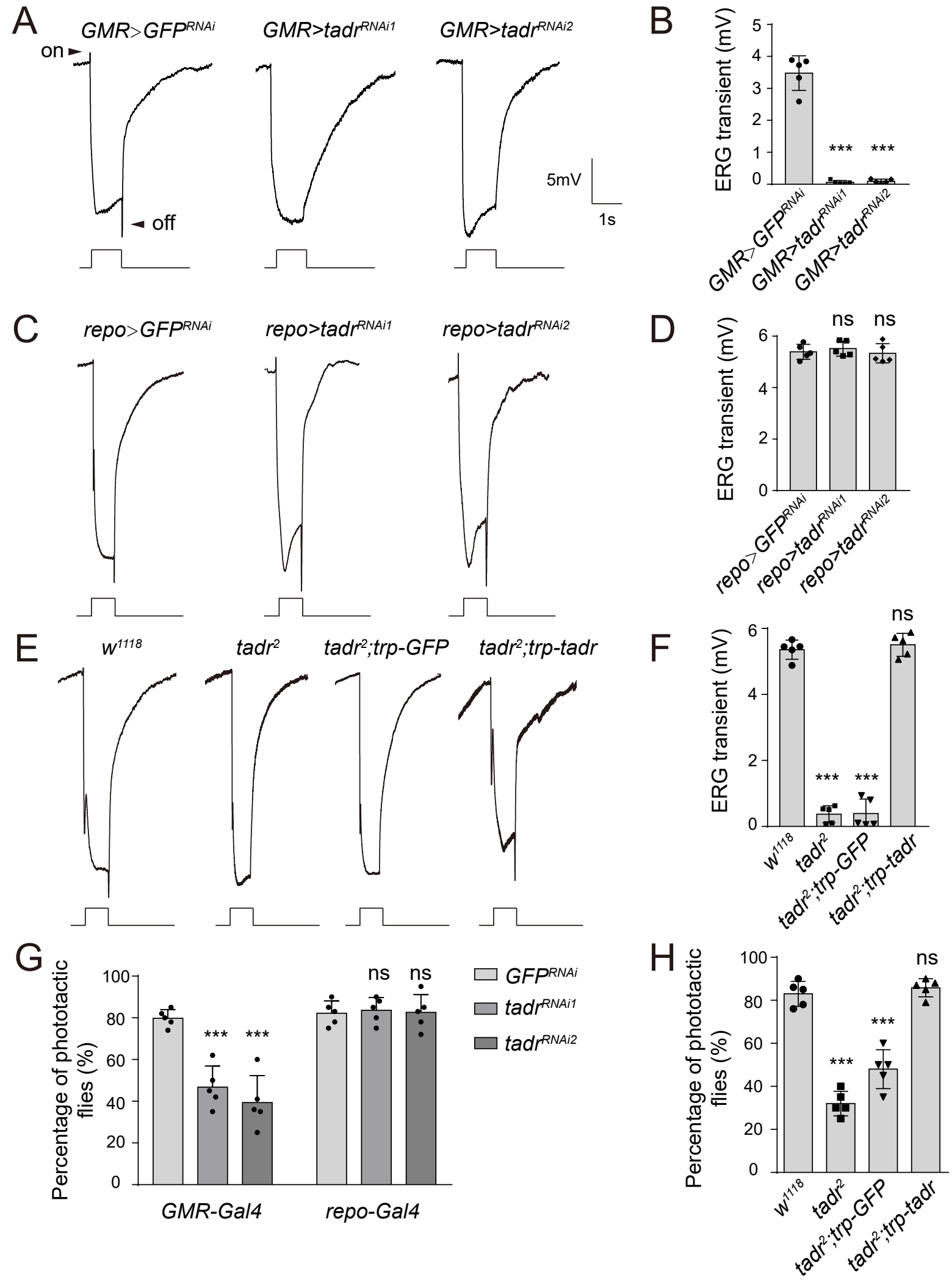
Figure 2-figure supplement 1
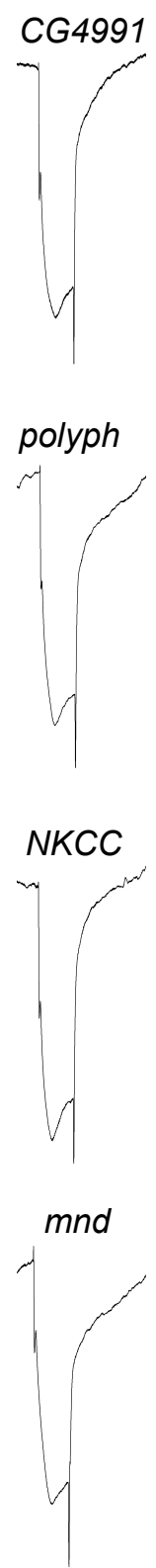

vGlut
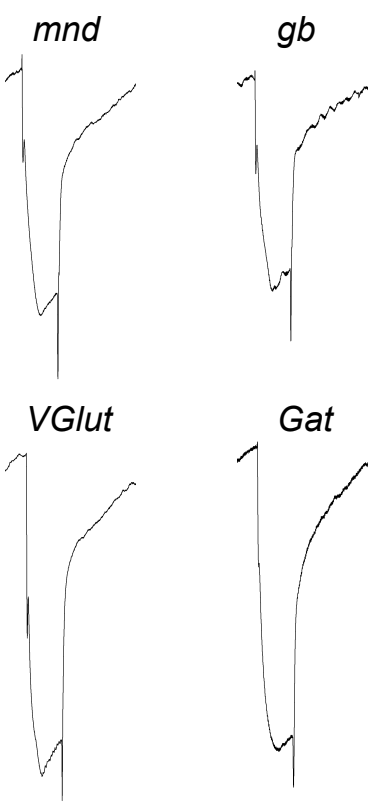

CG13784 CG32081

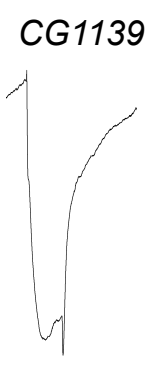

mah
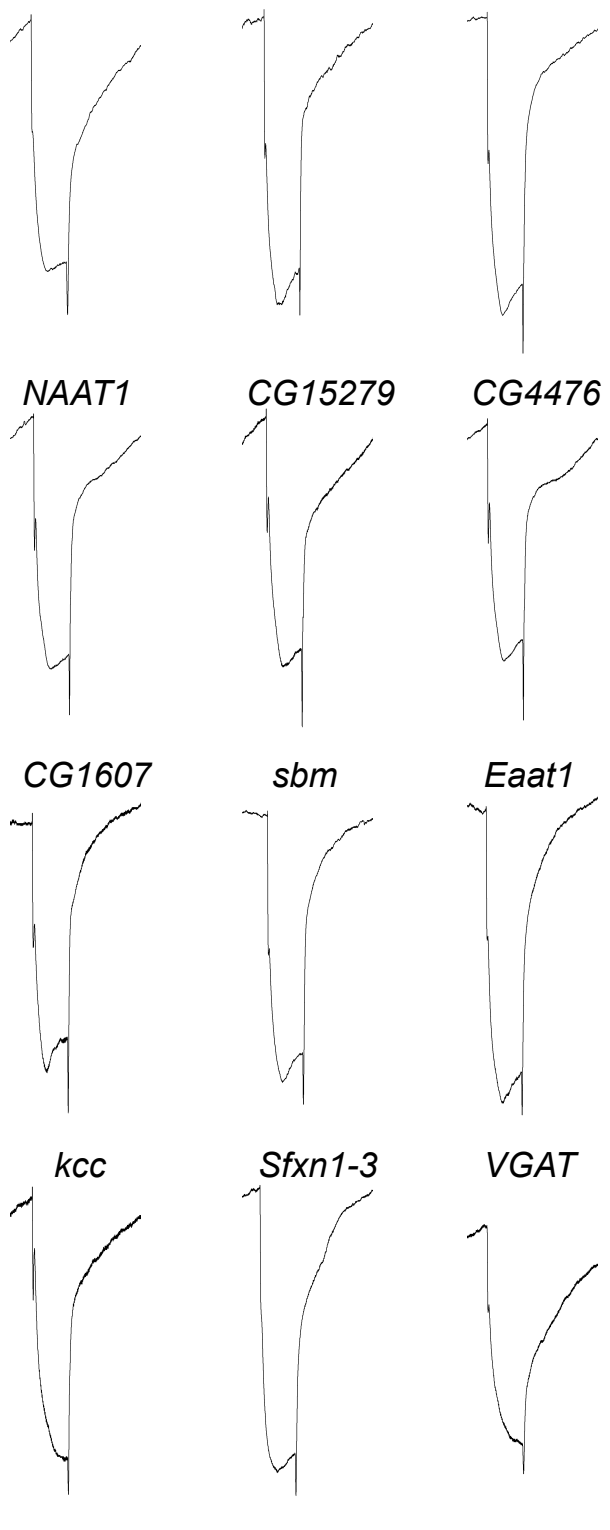

VGAT

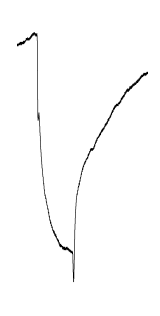

CG13248
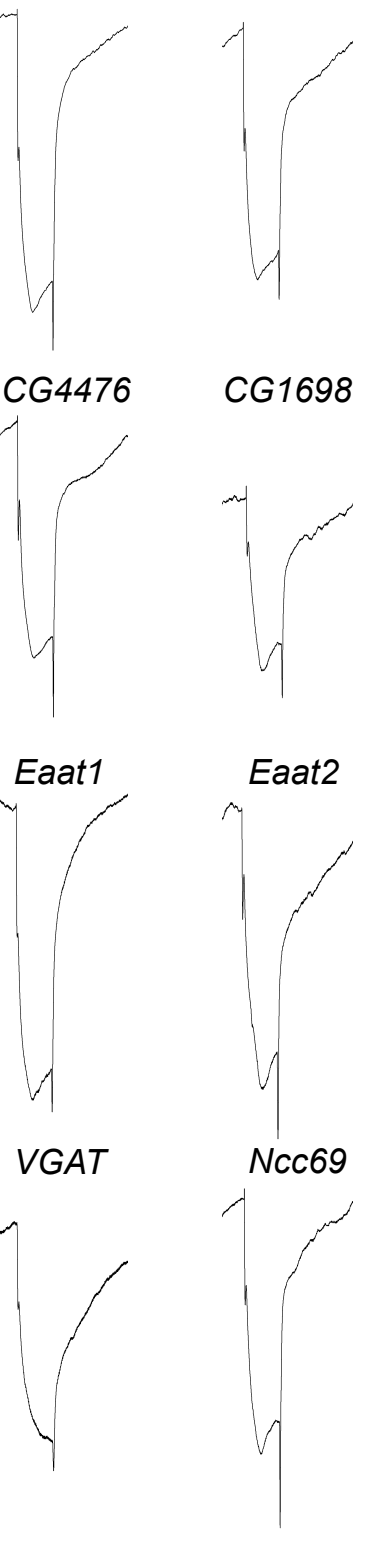
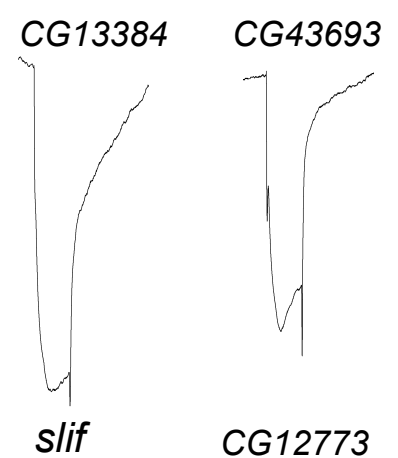

CG12773
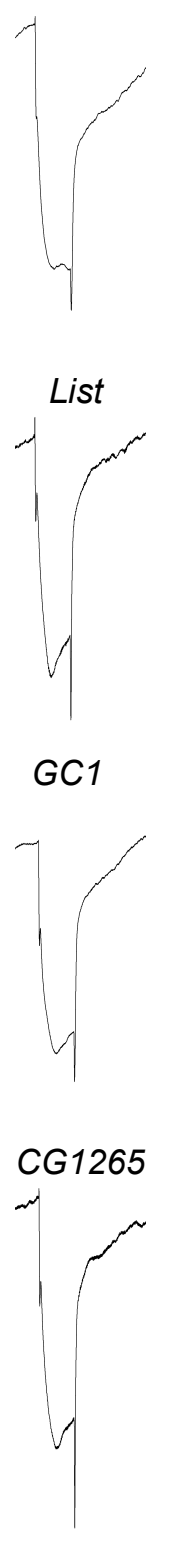
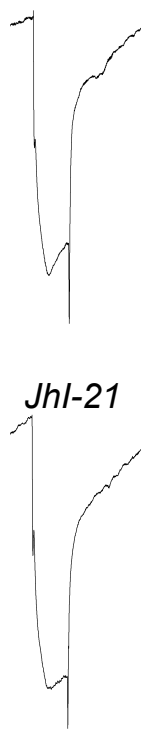

tadr

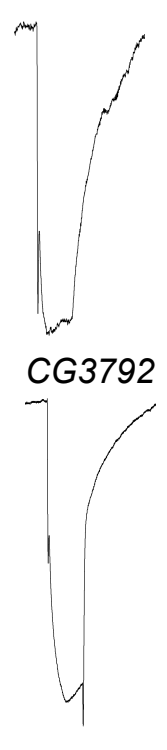


Figure 2-figure supplement 2

A
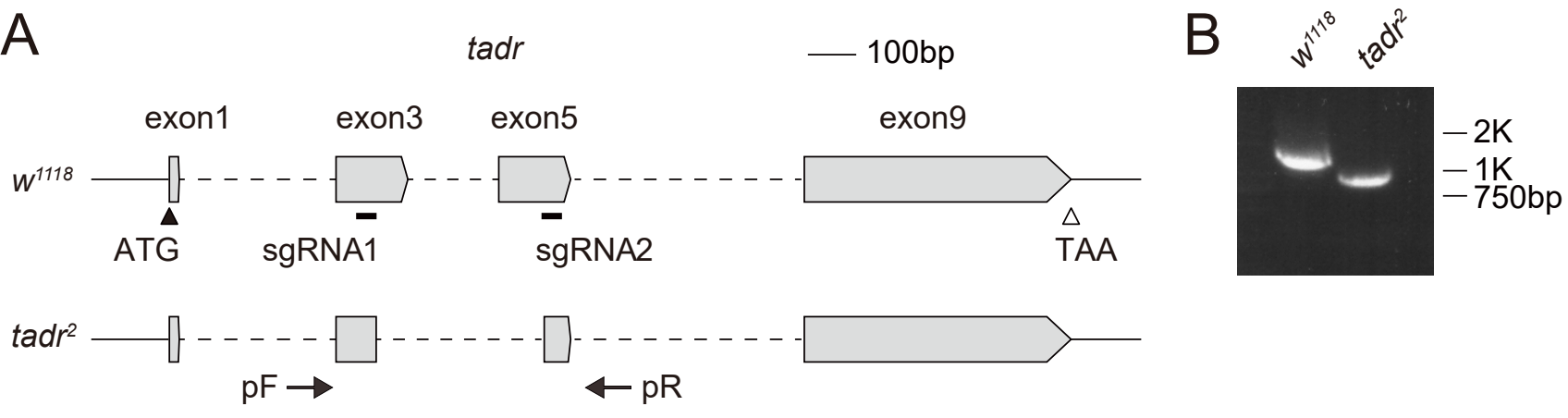

C

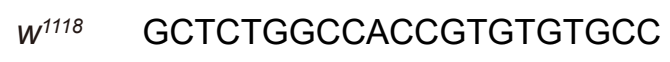

$\overline{\text { Ala }} \overline{\text { Leu }} \overline{\text { Ala }} \overline{\text { Thr }} \overline{\text { Val }} \overline{\text { Cys }} \overline{\text { Ala }}$

TGGTGGCGGGGCGACATG

$\overline{\operatorname{Trp}} \overline{\operatorname{Trp}} \overline{\operatorname{Arg}} \overline{\text { Gly }} \overline{\text { Asp }} \overline{\text { Met }}$

$244 \mathrm{bp}$

$t^{2} r^{2} \quad$ GCTCTGGCCACCGTGTGTGCC ................. GGCGGGGCGACATG ..........

$\overline{\text { Ala }} \overline{\text { Leu }} \overline{\text { Ala }} \overline{\text { Thr }} \overline{\text { Val }} \overline{\text { Cys }} \overline{\text { Ala }}$

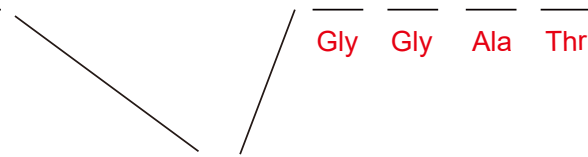

GCT CT GGCCACCGTGTGTGCCGGCGGGGCGACAT G

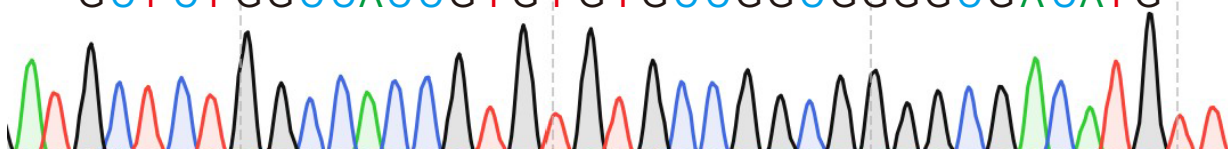


Figure 2-figure supplement 3

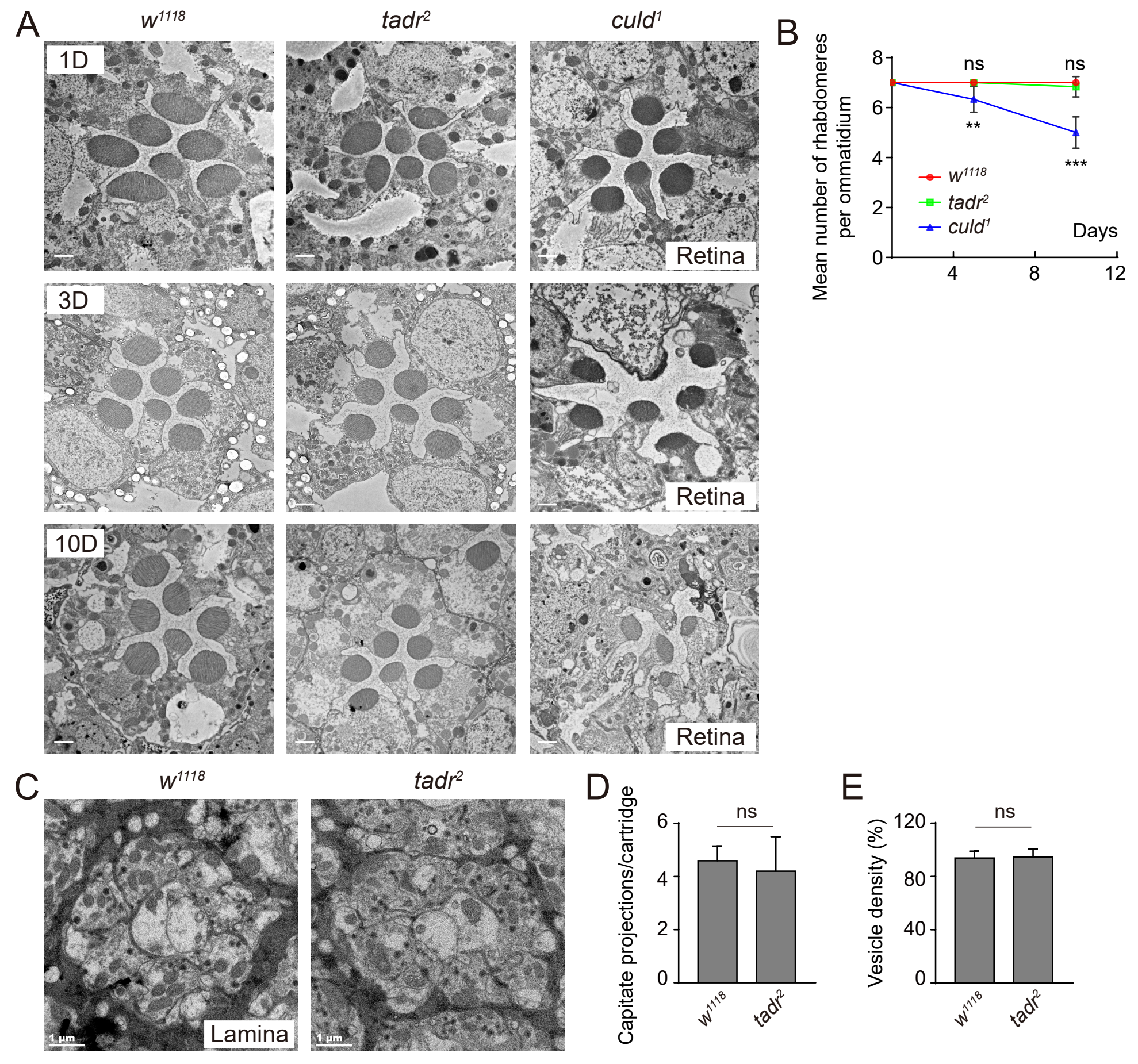


Figure 3
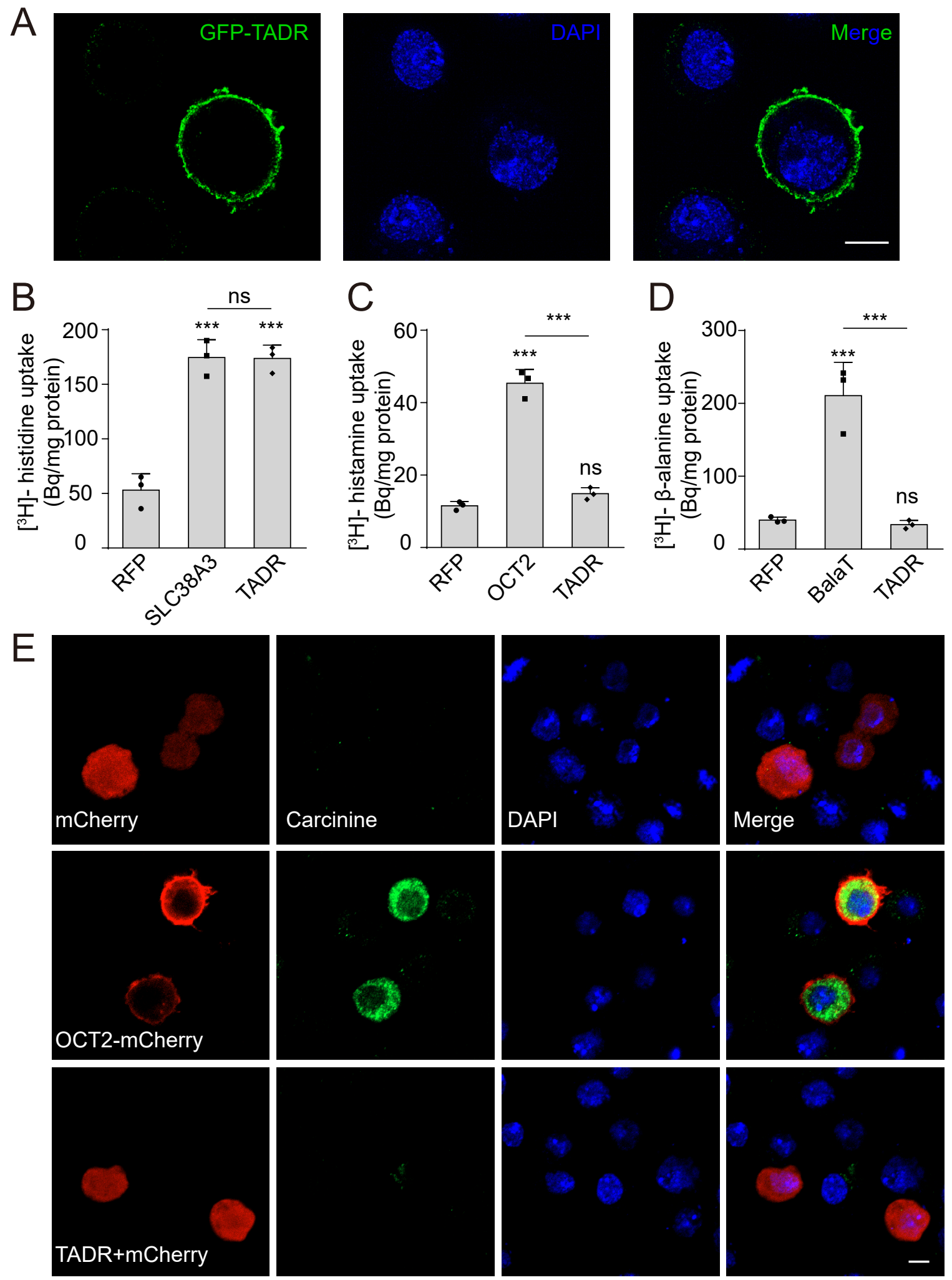
Figure 3-figure supplement 1

A
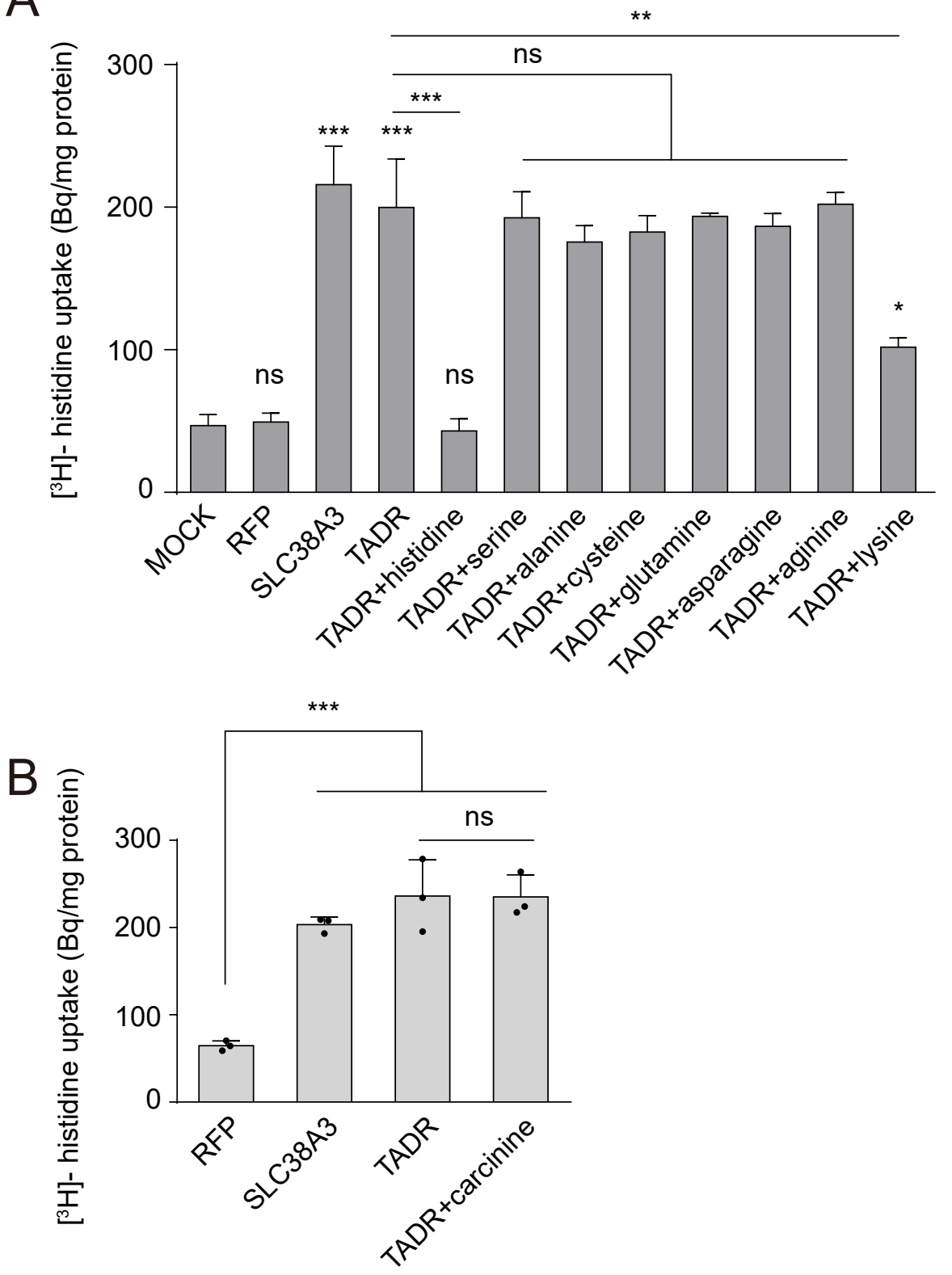
Figure 4

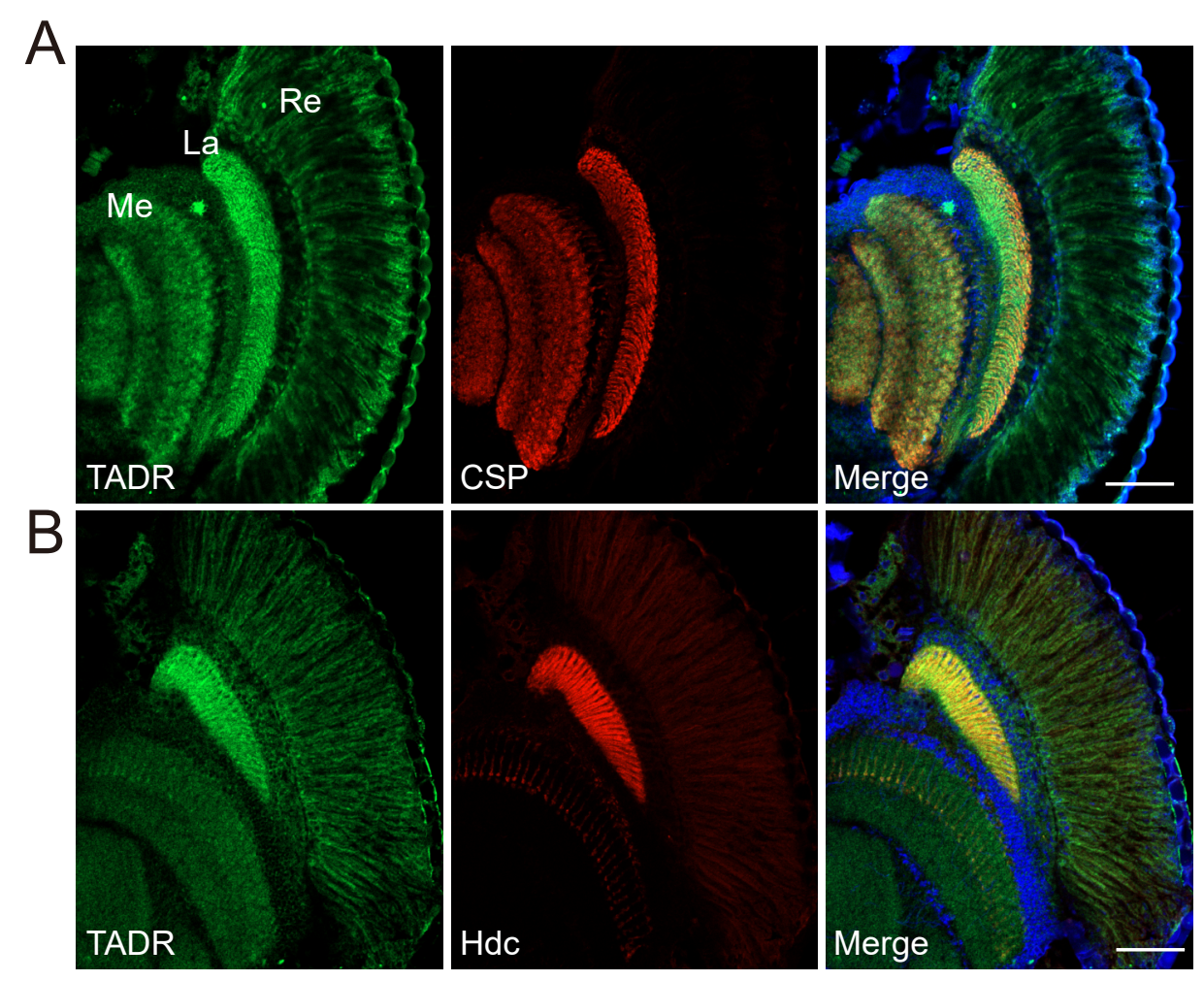


Figure 4-figure supplement 1

A

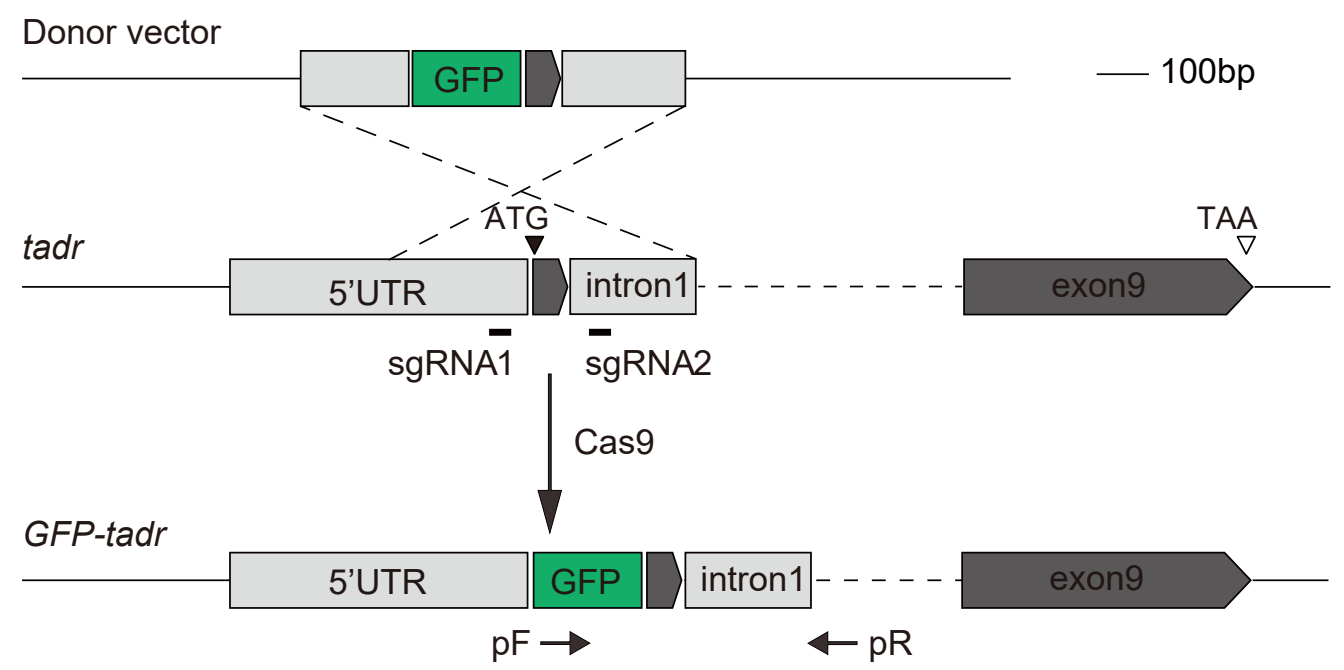

B
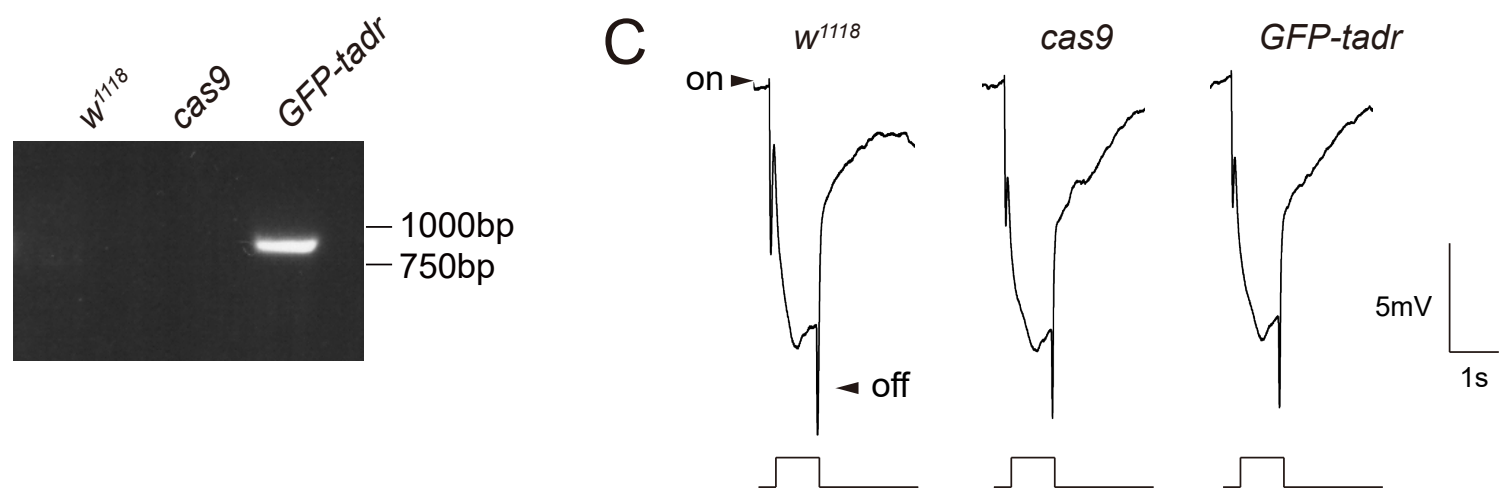
Figure 5

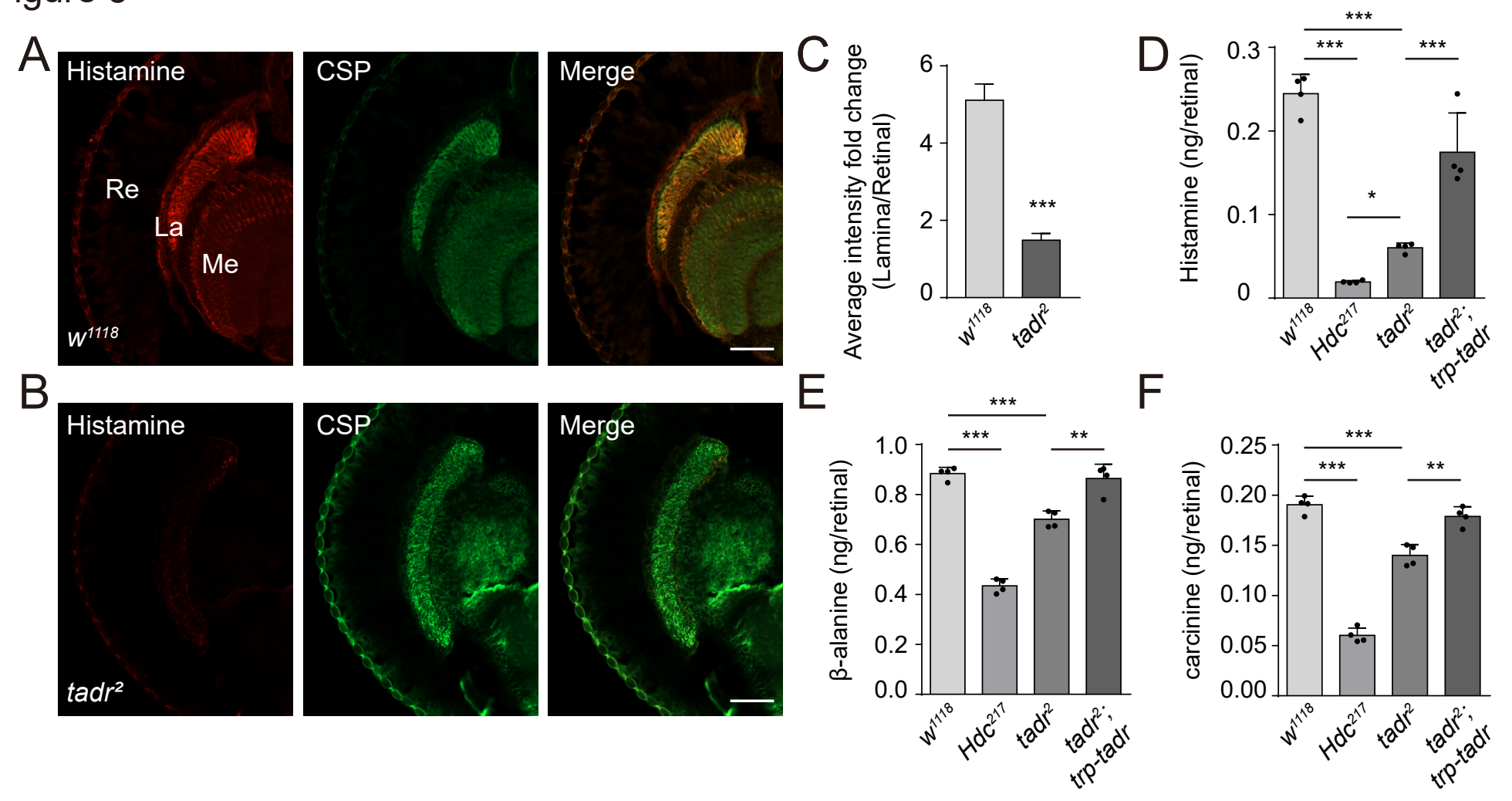


Figure 6
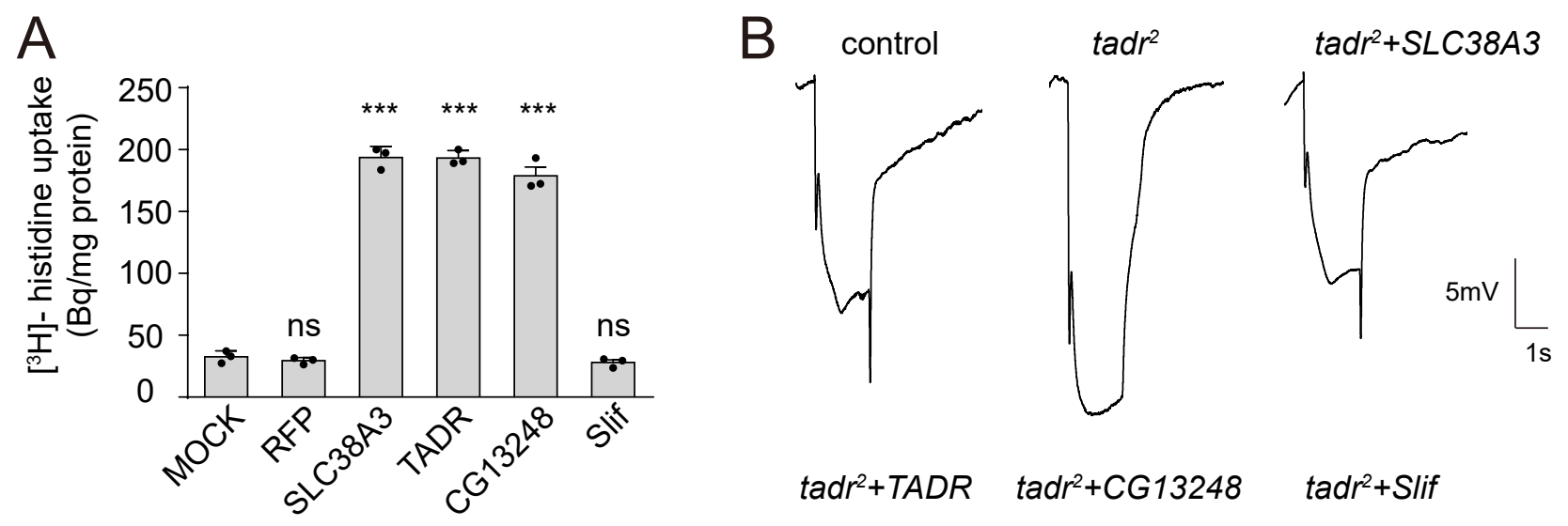

C

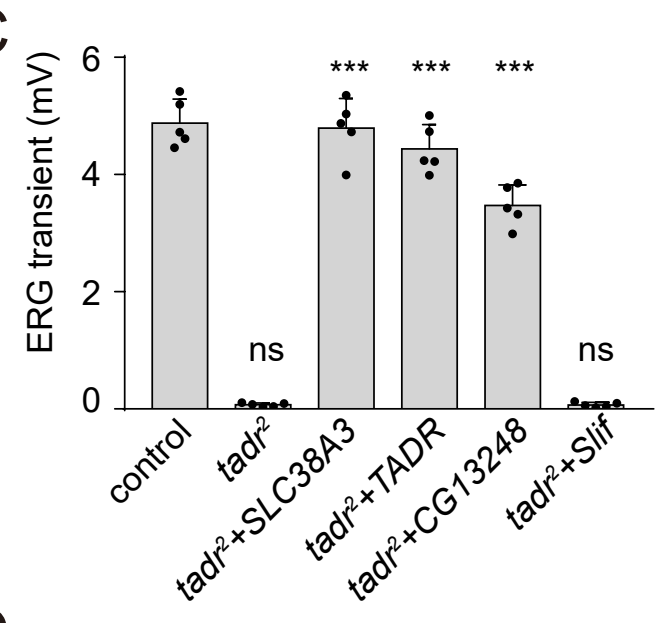

tadr $r^{2}+T A D R \quad t a d r^{2}+C G 13248 \quad t a d r^{2}+S l i f$

D

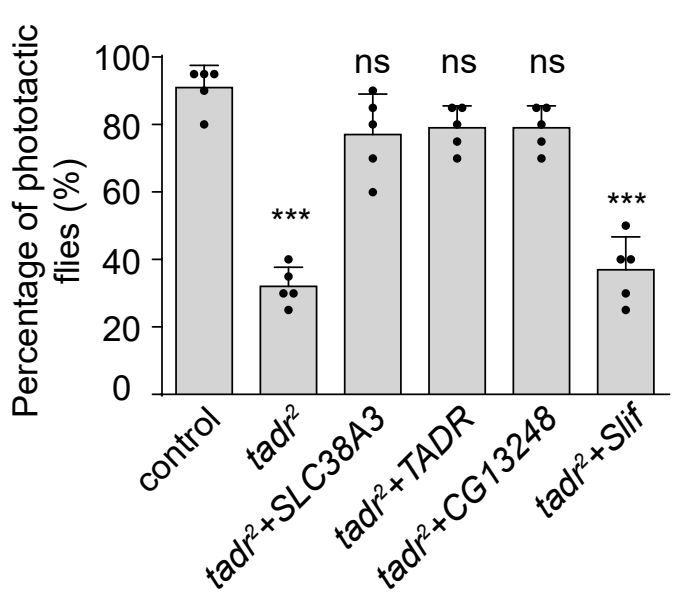

E
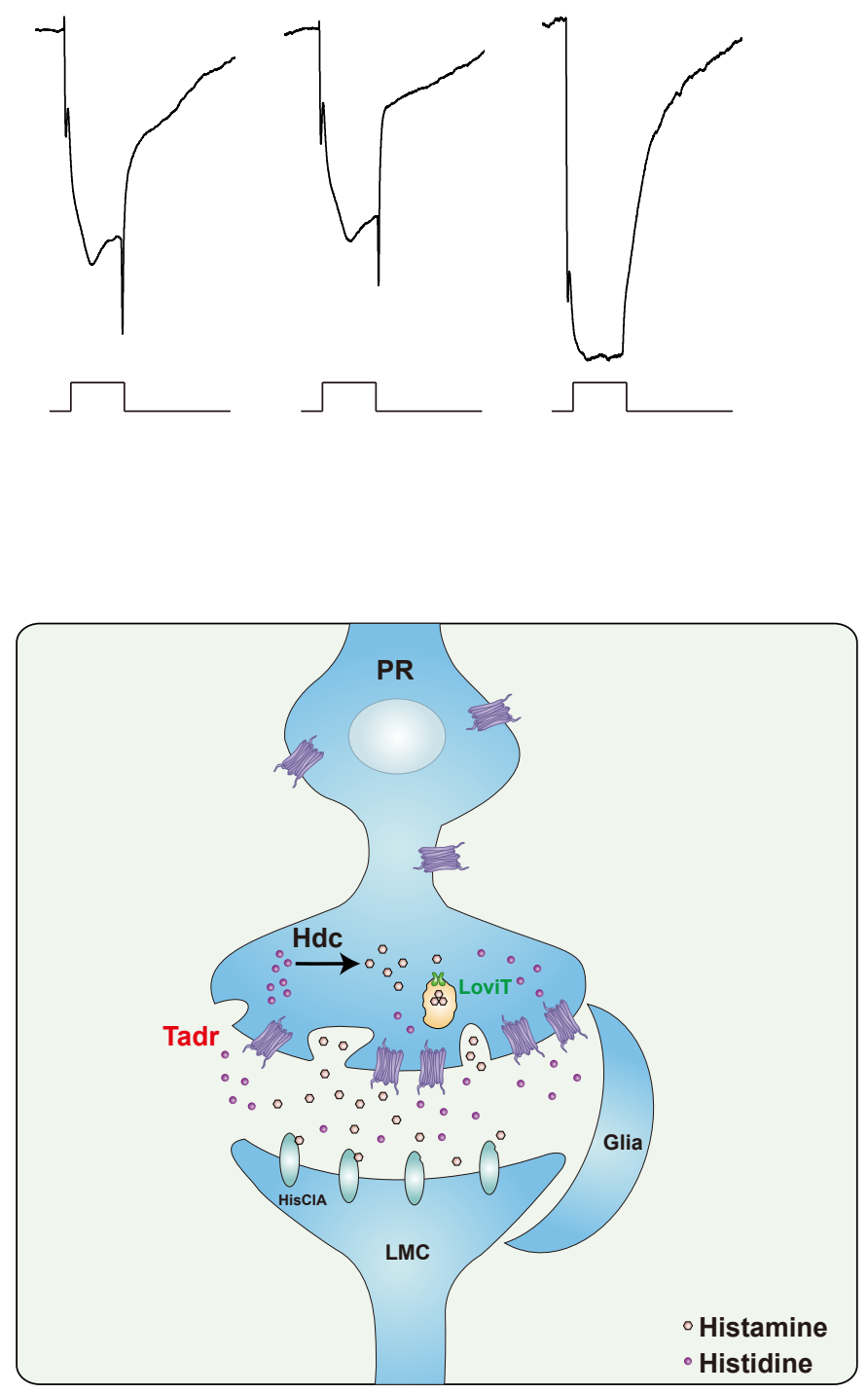
Figure 6-figure supplement 1

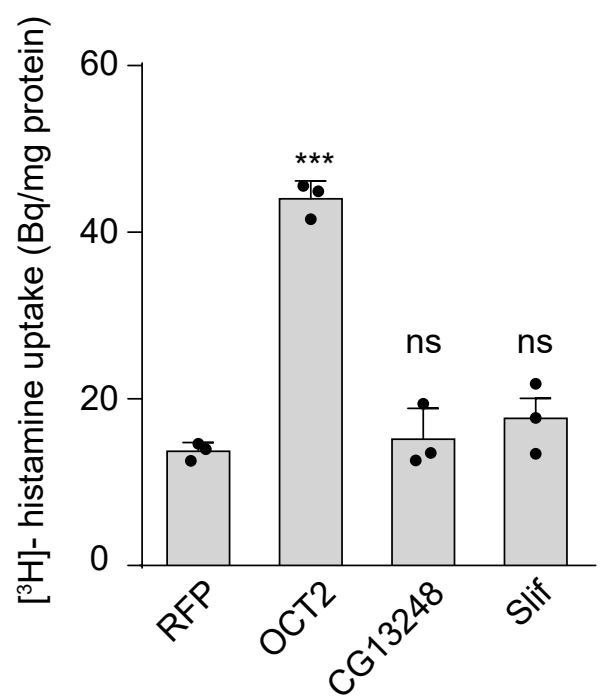

